\title{
Mg isotopic ratios in giant stars of the globular cluster NGC $6752^{\star}$
}

\author{
D. Yong ${ }^{1}$, F. Grundahl ${ }^{2}$, D. L. Lambert ${ }^{1}$, P. E. Nissen ${ }^{2}$, and M. D. Shetrone ${ }^{3}$ \\ 1 Department of Astronomy, University of Texas, Austin, TX 78712, USA \\ 2 Department of Physics and Astronomy, University of Aarhus, 8000 Aarhus C, Denmark \\ e-mail: fgj, pen@phys.au.dk \\ 3 McDonald Observatory, University of Texas, HC 75, Box 1337 MCD, Fort Davis, TX 79734, USA \\ e-mail: shetrone@astro.as.utexas.edu
}

Received 13 January 2003 / Accepted 27 February 2003

\begin{abstract}
Mg isotopic abundance ratios are measured in 20 bright red giants in globular cluster NGC 6752 based on very high-resolution $(R \sim 110000)$, high signal-to-noise spectra obtained with UVES on the VLT. There is a considerable spread in the ratio ${ }^{24} \mathrm{Mg}:{ }^{25} \mathrm{Mg}:{ }^{26} \mathrm{Mg}$ with values ranging from 53:9:39 to 83:10:7. We measured the abundances of $\mathrm{O}, \mathrm{Na}, \mathrm{Mg}, \mathrm{Al}$, and Fe combining our sample with 21 RGB bump stars (Grundahl et al. 2002). The abundances of the samples are consistent and exhibit the usual anticorrelations between $\mathrm{O}-\mathrm{Na}$ and $\mathrm{Mg}-\mathrm{Al}$. A positive correlation is found between ${ }^{26} \mathrm{Mg}$ and $\mathrm{Al}$, a mild anticorrelation is found between ${ }^{24} \mathrm{Mg}$ and $\mathrm{Al}$, while no correlation is found between ${ }^{25} \mathrm{Mg}$ and $\mathrm{Al}$. None of the elemental or isotopic abundances show a dependence on evolutionary status and, as shown by Gratton et al. (2001), the abundance variations exist even in main sequence stars. This strongly suggests that the star-to-star abundance variations are a result of varying degrees of pollution with intermediate mass AGB stars being likely polluters. Consideration of the extremes of the abundance variations provides the composition of the ambient material and the processed material. In the least contaminated stars (lowest $\mathrm{Na}$ and $\mathrm{Al}$ and highest $\mathrm{O}$ and $\mathrm{Mg}$ abundances), we infer a $\mathrm{Mg}$ isotopic ratio around 80:10:10 and a composition $([\mathrm{O} / \mathrm{Fe}],[\mathrm{Na} / \mathrm{Fe}],[\mathrm{Mg} / \mathrm{Fe}]$, $[\mathrm{Al} / \mathrm{Fe}]) \simeq(0.6,-0.1,0.5,0.0)$. In the most polluted stars, we find a $\mathrm{Mg}$ isotopic ratio around 60:10:30 and a composition $([\mathrm{O} / \mathrm{Fe}],[\mathrm{Na} / \mathrm{Fe}],[\mathrm{Mg} / \mathrm{Fe}],[\mathrm{Al} / \mathrm{Fe}]) \simeq(-0.1,0.6,0.3,1.2)$. Even for the least polluted stars, the abundances of ${ }^{25} \mathrm{Mg}$ and ${ }^{26} \mathrm{Mg}$ relative to ${ }^{24} \mathrm{Mg}$ are considerably higher than predicted for ejecta from $Z=0$ supernovae. Zero metallicity AGB stars may be responsible for these higher abundances. Our measured $\mathrm{Mg}$ isotopic ratios reveal another layer to the globular cluster star-to-star abundance variations that demands extensions of our present theoretical knowledge of stellar nucleosynthesis by giant stars.
\end{abstract}

Key words. galaxies: clusters: general - galaxies: clusters: individual: NGC 6752 - stars: abundances - stars: evolution stars: fundamental parameters

\section{Introduction}

Galactic globular clusters continue to play a central role in modern astrophysics for a variety of reasons. Firstly, as the oldest Galactic objects for which reliable ages have been determined (Vandenberg et al. 1996), they place a lower limit on the age of the universe. Secondly, besides the notable exceptions $\omega$ Cen and possibly M 22 (Lehnert et al. 1991), all stars within a given cluster have the same iron abundance within a narrow range though the metallicity varies considerably from cluster to cluster. This is surely a vital clue regarding the origins of globular clusters that has received relatively little theoretical attention (Brown et al. 1991, 1995; Lin \& Murray 1996; Parmentier et al. 1999; Nakasato et al. 2000). Finally, every

Send offprint requests to: $\mathrm{D}$. Yong,

e-mail: tofu,dll@astro.as.utexas.edu

* Based on observations obtained with the ESO Very Large Telescope UVES spectrograph for programmes 67.D-0145 and 65.L-0165. well studied Galactic globular cluster shows star-to-star abundance variations for light elements $(\mathrm{C}, \mathrm{N}, \mathrm{O}, \mathrm{Na}, \mathrm{Mg}$, and $\mathrm{Al})$. Although the amplitude of the variations may differ from cluster to cluster, there is a common pattern: the abundances of $\mathrm{C}$ and $\mathrm{O}$ are low when $\mathrm{N}$ is high, $\mathrm{O}$ and $\mathrm{Na}$ are anticorrelated as are $\mathrm{Mg}$ and $\mathrm{Al}$.

Two scenarios compete for priority in accounting for the star-to-star abundance variations: the so-called evolutionary and primordial scenarios. The evolutionary scenario supposes that the variations arise when a star becomes a red giant with a convective envelope that may tap deep layers where $\mathrm{H}$-burning occurs through the $\mathrm{CNO}-$ cycles, and possibly the $\mathrm{Ne}-\mathrm{Na}$ and $\mathrm{Mg}-\mathrm{Al}$ chains. Standard models of red giants do not predict the required deep extension of the convective envelope but its invocation is not totally physically implausible. If the extension were controlled by (say) the rotation of the star, a star-to star abundance variation could occur among red giants. A primordial scenario places the first appearance of the abundance variations in main sequence stars. One possibility is that the 
cluster gas was not of a homogeneous composition when the stars formed. A second possibility is that stars may have formed with identical or nearly identical compositions but subsequent accretion of gas ejected by evolved stars resulted in the star-tostar abundance variations.

Abundance variations were discovered first among cluster red giants for obvious reasons. Later, variations were found to be present among subgiants and even in main sequence stars where an evolutionary scenario is excluded on the firm ground that the internal temperatures required for hydrostatic equilibrium are too low for the $\mathrm{Ne}-\mathrm{Na}$ and $\mathrm{Mg}-\mathrm{Al}$ chains to run by proton captures. The pertinent observations involve variously: narrow band photometry and low or high resolution spectroscopy including those reported initially by Hesser (1978), Hesser \& Bell (1980), and Bell et al. (1983) for 47 Tuc and more recently by Cannon et al. (1998), Cohen (1999), Gratton et al. (2001), Cohen et al. (2002), Briley et al. (2002), and Ramírez \& Cohen (2003) for a variety of clusters. In some or all clusters, primordial variations for some elements may be supplemented by an evolutionary component. For example, the carbon abundances are correlated with luminosity of the red giants of globular clusters M 4, M 22, NGC 6752, $\omega$ Cen, and 47 Tuc (Smith \& Suntzeff 1989; Brown \& Wallerstein 1989; Suntzeff \& Smith 1991; Cannon et al. 2002). It has also been suggested that variations in the He abundance may play a role in the morphology of the horizontal branch (D'Antona et al. 2002).

In this paper, we examine a novel aspect of the star-to-star abundance variations among red giants of the globular cluster NGC 6752. This is the cluster in which Cottrell \& Da Costa (1981) discovered the correlated $\mathrm{Na}$ and $\mathrm{Al}$ enhancements in CN-strong giants. This cluster with M 13 (Kraft et al. 1997) shows the largest amplitude in the star-to-star abundance variations of all the well-studied globular clusters. Suntzeff \& Smith (1991) through a combination of low-resolution optical and infrared spectra found $\mathrm{C}$ and $\mathrm{N}$ abundance variations extending from the main sequence to the red giant stars of NGC 6752. Among red giants, the carbon abundance was shown to decline with increasing luminosity, a sign of an evolutionary contribution to the predominant primordial scenario for explaining the abundance variations. Recently, Gratton et al. (2001) reported finding the $\mathrm{O}-\mathrm{Na}$ and $\mathrm{Mg}-\mathrm{Al}$ anticorrelations among stars at the main sequence turn-off and Grundahl et al. (2002) obtained similar results for stars on the red giant branch around the bump on this branch. Our extension involves the measurement of magnesium isotopic ratios for a sample of the most luminous giants. We also determine the $\mathrm{O}, \mathrm{Na}, \mathrm{Mg}, \mathrm{Al}$, and $\mathrm{Fe}$ abundances. Our goal is to examine the primordial and evolutionary scenarios in light of the $\mathrm{Mg}$ isotopic abundances.

The first attempt to measure $\mathrm{Mg}$ isotopic ratios for globular cluster giants was by Shetrone (1996a,b) for a few giants in M 13, a cluster with both a similar metallicity and spread in light element abundances to NGC 6752. With the VLT and UVES, it is possible to obtain the high quality spectra necessary for measuring the $\mathrm{Mg}$ isotopic ratios in a sample of the brightest giants in NGC 6752. Whereas Shetrone was unable to separate the contribution of ${ }^{25} \mathrm{Mg}$ from ${ }^{26} \mathrm{Mg}$, we will make this important distinction. In Sect. 2 the sample selection and data acquisition are covered. In Sect. 3 the derivation of the stellar parameters is presented. The elemental abundances are determined in Sect. 4 and $\mathrm{Mg}$ isotopic ratios in Sect. 5. The discussion is in Sect. 6 and concluding remarks are given in Sect. 7.

\section{Data acquisition}

\subsection{Target selection}

The targets for this study were selected from the uvby photometry of NGC 6752 in Grundahl et al. (1999). We selected the stars such that there would be no detected neighbours within the entrance aperture $(1.5 \times 2.0 \mathrm{arcsec})$ of the UVES image slicer in order to minimize contamination. The cluster colourmagnitude diagram (CMD) shown in Fig. 1 was used to select the best first ascent RGB stars. The sample is free from AGB contamination fainter than $V=11.4$. Brighter than this the contamination is very unlikely since the evolutionary timescale on the RGB is much slower than for AGB stars.

Three additional stars (B 702, B 708, A88) used in the analysis are not shown in Fig. 1. All 3 stars were analyzed by Shetrone (1998). Reobservations were made for B 702 and A88 and all three stars were reanalyzed. The $V$ magnitudes and $B-V$ colours place these stars at the tip of the RGB.

\subsection{Observations and reduction}

The observations for this programme were carried out with the UVES instrument on the ESO VLT UT2 telescope using the red arm in the standard setting of center $580 \mathrm{~nm}$ and cross disperser \#3. In order to separate the $\mathrm{Mg}$ isotopic components, the highest possible spectrograph resolution $R=110000$ was used for all observations. This corresponds to a slit width of 0 ". 3 , so we used the image slicer (\#3) to minimize light losses due to seeing. Our programme stars were observed in service mode during the summer of 2001. The integration time for each star was split in three to be able to remove the effects of cosmic ray hits on the CCD detector and the total exposure times ranged between 3600 and $5400 \mathrm{~s}$, depending on the brightness of the target star. Our spectra covered the wavelength region between $4735 \AA$ and $6830 \AA$ with a small gap between $5800 \AA$ and $5825 \AA$ due to the space between the two CCDs in the UVES camera. The signal-to-noise ranged from 250 to 150 per pixel. In addition, we also observed the hot star HR 6788 in order to use it for removal of telluric features (using telluric in IRAF $^{1}$ ) near the [OI] line at $6300 \AA$.

Standard IRAF procedures were used for bias subtraction, correction for interorder background, flat fielding and extraction of spectra. The total width of the 5 slices in each order was 10 arcsec and for each wavelength bin we summed the flux over all corresponding pixels. Wavelength calibration was carried out on a nightly basis using Th-Ar spectra.

\footnotetext{
${ }^{1}$ IRAF is distributed by the National Optical Astronomy Observatories, which are operated by the Association of Universities for Research in Astronomy, Inc., under cooperative agreement with the National Science Foundation.
} 


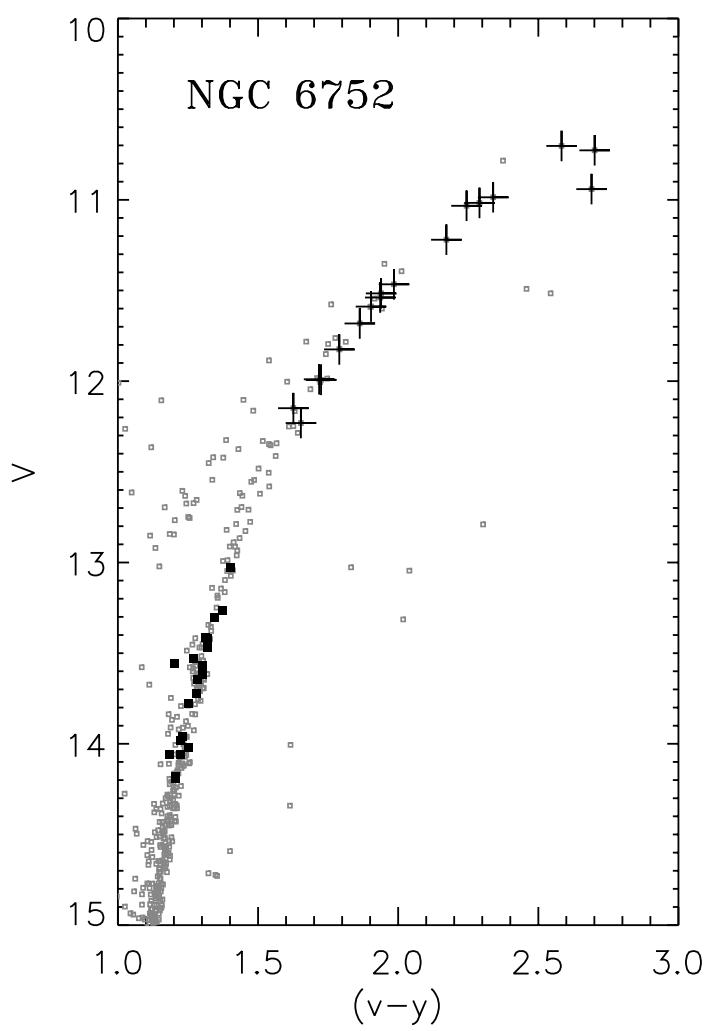

Fig. 1. The colour-magnitude diagram from NGC 6752. The large plus symbols represent the bright red giants reported here while the filled black squares represent the RGB bump stars observed under ESO programme 65.L-0165 and reported by Grundahl et al. (2002).

By using the image slicer in UVES the sky spectrum could not be measured simultaneously with the star spectrum. We note, however, that even for the faintest stars in our sample, $V \approx 12$, observed in full Moon periods the sky signal integrated over the $10 \times 0.3$ arcsec slit is less than $0.3 \%$ of the star signal in the spectral region from $4800 \AA$ to $6800 \AA$. Hence, the sky contribution can be neglected.

For the three additional stars, A88 and B 708 were observed on the VLT in service mode during the summer of 2000 with $R=110000$ and signal-to-noise around 100. B 702 was observed with the ESO CAT with $R=80000$, for details about this observation see Shetrone (1998).

\section{Stellar parameters}

\section{1. $T_{\text {eff }}$ and $\log g$}

For determining the effective temperature and surface gravity of our target stars we used the same procedure as described by Grundahl et al. (2002), which we shall briefly repeat here. Temperatures were derived from the uvby photometry using the $(b-y)$ index and the calibration by Alonso et al. (1999), adopting $E(B-V)=0.04$ (Harris 1996) for the cluster reddening. To estimate the surface gravity we adopted an apparent distance modulus of $(m-M)_{V}=13.30$ for the cluster and a stellar mass of $0.84 M_{\odot}$. The bolometric corrections were taken from

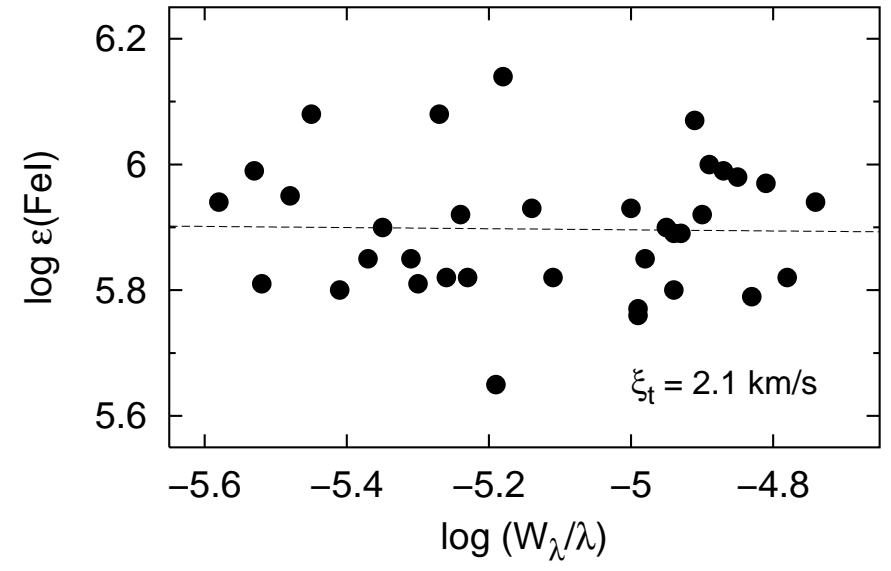

Fig. 2. The abundances derived from individual Fe I lines plotted against the reduced equivalent width $\left(W_{\lambda} / \lambda\right)$ for star NGC 6752-mg6. The microturbulence, $\xi_{\mathrm{t}}$, is set by the requirement that the abundances (derived from individual Fe I lines) show no trend against $\left(W_{\lambda} / \lambda\right)$. The dotted line represents the linear least squares fit to the data.

a $14 \mathrm{Gyr}$ isochrone with $[\mathrm{Fe} / \mathrm{H}]=-1.54$ from VandenBerg et al. (2000). The stellar parameters are presented in Table 1. We found that changes of \pm 0.1 in distance modulus led to changes of -0.04 dex in the derived gravity and changes of \pm 0.02 in $(b-y)$ (reddening and calibration uncertainty) resulted in errors in the temperature of approximately $40 \mathrm{~K}$ (average value for the 17 stars). From Table 2 we therefore see that the uncertainty of distance modulus and cluster photometry have very little impact on our derived results.

\subsection{Microturbulence}

For each star, we determined the abundance of key elements beginning with the iron abundance as the canonical measure of metallicity. A model atmosphere was taken from the Kurucz (1993) LTE stellar atmosphere grid. We interpolated within the grid when necessary to get a model with the required $T_{\text {eff }}, \log g$, and $[\mathrm{Fe} / \mathrm{H}]$. The model was used with the LTE stellar line analysis program Moog (Sneden 1973). The equivalent width of a line was measured using IRAF where in general a Gaussian profile was fit to an observed profile. To complete the abundance analysis, an estimate of the microturbulence is needed. This was determined from Fe I lines.

A selection of $31 \mathrm{Fe}$ I and 8 Fe II lines was measured. The $g f$-values were taken mostly from Lambert et al. (1996) and a small number from a list compiled by Luck (1993, private communication). The microturbulence $\left(\xi_{\mathrm{t}}\right.$ in $\left.\mathrm{km} \mathrm{s}^{-1}\right)$ was determined in the usual way by insisting that the Fe abundance from $\mathrm{Fe}$ I lines be independent of their equivalent width $\left(W_{\lambda}\right)$. An example of the abundance versus $W_{\lambda}$ plot is shown in Fig. 2. The derived $\xi_{\mathrm{t}}$ range from about $2.7 \mathrm{~km} \mathrm{~s}^{-1}$ for the stars at the tip of the giant branch to $1.8 \mathrm{~km} \mathrm{~s}^{-1}$ for our faintest stars which are 1.5 mag below the tip. The measurement uncertainty is typically about $\pm 0.2 \mathrm{~km} \mathrm{~s}^{-1}$. 
Table 1. Stellar parameters and elemental abundances for program stars.

\begin{tabular}{|c|c|c|c|c|c|c|c|c|c|c|c|c|c|c|c|}
\hline \multirow[t]{2}{*}{ Namel } & \multirow[t]{2}{*}{ Name2 } & \multirow{2}{*}{$\begin{array}{c}\text { RA } \\
(2000)\end{array}$} & \multirow{2}{*}{$\begin{array}{c}\text { Dec } \\
(2000)\end{array}$} & \multirow[t]{2}{*}{ V } & \multirow{2}{*}{$\begin{array}{l}T_{\text {eff }} \\
(\mathrm{K})\end{array}$} & \multirow{2}{*}{$\begin{array}{c}\log g \\
\left(\mathrm{~cm} \mathrm{~s}^{-2}\right)\end{array}$} & \multirow{2}{*}{\multicolumn{2}{|c|}{$\begin{array}{c}\xi_{\mathrm{t}} \text { Macro } \\
\left(\mathrm{km} \mathrm{s}^{-1}\right)\end{array}$}} & $\mathrm{Fe}$ I & Fe II & $\mathrm{Fe}^{a}$ & $\mathrm{O}$ & $\mathrm{Na}$ & $\mathrm{Mg}$ & $\mathrm{Al}$ \\
\hline & & & & & & & & & \multicolumn{7}{|c|}{$\log \epsilon($ Species $)$} \\
\hline NGC 6752-mg0 & PD1 & 19:10:58 & $-59: 58: 07$ & 10.70 & 3928 & 0.26 & 2.7 & 5.00 & 5.85 & 6.00 & 5.88 & 7.22 & 5.36 & 6.42 & 5.91 \\
\hline NGC 6752-mg1 & B1630 & 19:11:11 & $-59: 59: 51$ & 10.73 & 3900 & 0.24 & 2.7 & 4.75 & 5.87 & 6.05 & 5.90 & 7.53 & 5.09 & 6.42 & 5.65 \\
\hline NGC 6752-mg2 & B3589 & $19: 10: 32$ & $-59: 57: 01$ & 10.94 & 3894 & 0.33 & 2.5 & 6.00 & 5.87 & 6.09 & 5.91 & 7.63 & 4.91 & 6.44 & 5.60 \\
\hline NGC 6752-mg3 & B1416 & 19:11:17 & $-60: 03: 10$ & 10.99 & 4050 & 0.50 & 2.2 & 5.50 & 5.89 & 5.94 & 5.90 & 7.54 & 4.93 & 6.46 & 5.60 \\
\hline NGC 6752-mg4 & $\ldots$ & $19: 10: 43$ & $-59: 59: 54$ & 11.02 & 4065 & 0.53 & 2.2 & 5.50 & 5.89 & 5.96 & 5.90 & 7.45 & 5.00 & 6.43 & 5.73 \\
\hline NGC 6752-mg5 & PD2 & 19:10:49 & $-59: 59: 34$ & 11.03 & 4100 & 0.56 & 2.1 & 5.00 & 5.90 & 5.96 & 5.91 & 7.50 & 5.04 & 6.48 & 5.57 \\
\hline NGC 6752-mg6 & В2113 & 19:11:03 & $-60: 01: 43$ & 11.22 & 4154 & 0.68 & 2.1 & 4.50 & 5.90 & 5.94 & 5.91 & 7.68 & 4.85 & 6.51 & 5.40 \\
\hline NGC 6752-mg $8^{b}$ & $\ldots$ & 19:10:38 & $-60: 04: 10$ & 11.47 & 4250 & 0.80 & 2.0 & 5.00 & 5.82 & 5.84 & 5.82 & 7.39 & 4.97 & 6.38 & 5.57 \\
\hline NGC 6752-mg9 & B3169 & 19:10:40 & $-59: 58: 14$ & 11.52 & 4288 & 0.91 & 1.9 & 5.00 & 5.87 & 5.86 & 5.87 & 7.51 & 4.96 & 6.44 & 5.60 \\
\hline NGC 6752-mg 10 & В2575 & 19:10:54 & $-59: 57: 14$ & 11.54 & 4264 & 0.90 & 1.8 & 4.75 & 5.86 & 5.90 & 5.87 & 7.48 & 4.96 & 6.44 & 5.61 \\
\hline NGC 6752-mg12 & $\ldots$ & 19:10:58 & $-59: 57: 04$ & 11.59 & 4286 & 0.94 & 1.8 & 5.25 & 5.87 & 5.92 & 5.88 & 7.71 & 4.60 & 6.42 & 4.92 \\
\hline NGC 6752-mg15 & B2196 & 19:11:01 & $-59: 57: 18$ & 11.68 & 4354 & 1.02 & 1.9 & 5.25 & 5.90 & 5.89 & 5.90 & 7.47 & 5.02 & 6.48 & 5.55 \\
\hline NGC 6752-mg 18 & B1518 & 19:11:15 & $-60: 00: 29$ & 11.83 & 4398 & 1.11 & 1.8 & 4.75 & 5.90 & 5.89 & 5.90 & 7.53 & 4.90 & 6.45 & 5.42 \\
\hline NGC 6752-mg21 & B3805 & 19:10:28 & $-59: 59: 49$ & 11.99 & 4429 & 1.20 & 1.8 & 5.25 & 5.90 & 5.92 & 5.90 & 7.08 & 5.28 & 6.38 & 6.01 \\
\hline NGC 6752-mg22 & В2580 & 19:10:54 & $-60: 02: 05$ & 11.99 & 4436 & 1.20 & 1.8 & 5.00 & 5.89 & 5.91 & 5.89 & 7.25 & 5.33 & 6.44 & 5.82 \\
\hline NGC 6752-mg24 & B1285 & 19:11:19 & $-60: 00: 31$ & 12.15 & 4511 & 1.31 & 1.9 & 5.00 & 5.88 & 5.84 & 5.87 & 7.69 & 4.59 & 6.43 & 4.95 \\
\hline NGC 6752-mg25 & В2892 & 19:10:46 & $-59: 56: 22$ & 12.23 & 4489 & 1.33 & 1.7 & 5.25 & 5.90 & 5.92 & 5.90 & 7.66 & 4.85 & 6.49 & 5.34 \\
\hline NGC $6752-702^{b}$ & B702 & 19:11:31 & $-59: 54: 33$ & 10.83 & 4050 & 0.50 & 2.1 & 5.50 & 5.90 & 5.98 & 5.92 & $\ldots$ & 5.17 & 6.28 & 6.05 \\
\hline NGC $6752-708^{b}$ & B708 & 19:11:31 & $-59: 54: 33$ & 10.58 & 4050 & 0.25 & 2.2 & $11.00^{d}$ & 5.85 & 5.95 & 5.87 & 7.44 & 4.91 & 6.41 & 5.52 \\
\hline NGC $6752-\mathrm{A} 88^{b}$ & A 88 & 19:10:40 & $-59: 54: 33$ & 10.52 & 4080 & 0.55 & 2.4 & 5.50 & 5.88 & 5.95 & 5.90 & 7.46 & 5.01 & 6.38 & 5.95 \\
\hline HD $103036^{c}$ & & & & 8.18 & 4200 & 0.10 & 3.1 & 7.25 & 5.73 & 5.69 & 5.72 & 7.54 & 4.71 & 6.33 & 5.08 \\
\hline HD $141531^{c}$ & & & & 9.15 & 4273 & 0.80 & 1.9 & 5.50 & 5.75 & 5.88 & 5.78 & 7.49 & 4.33 & 6.31 & 4.74 \\
\hline
\end{tabular}

$a$ Weighted mean of Fe I and Fe II.

$b$ Spectroscopically determined stellar parameters.

$c$ Comparison field stars.

${ }^{d}$ This high value is due to the lower resolving power.

Note. - PD1 and PD2 are from Penny \& Dickens (1986), the B xxxx names are from Buonanno et al. (1986), and A 88 is from Alcaino (1972).

\subsection{The iron abundance}

The Fe abundances from the Fe I lines are but weakly dependent on the derived $\xi_{\mathrm{t}}$ because the set of Fe I lines contains an ample number of weak lines. These abundances show a dispersion attributable to measurement errors. The mean abundance is $\log \epsilon(\mathrm{Fe})=5.89 \pm 0.01(\sigma=0.02)$ where the standard deviation is consistent with all stars having the same abundance. Determination of the Fe abundance from the smaller set of Fe II lines offers a check on the adopted stellar parameters and assumptions underlying the model atmospheres and the line analysis. The Fe II and Fe I lines give the same abundance for the warmer stars $\left(T_{\text {eff }} \geq 4200 \mathrm{~K}\right)$ but cooler stars show a discrepancy which increases with decreasing $T_{\text {eff }}$ (Fig. 3). The coolest giants show an Fe abundance about 0.2 dex larger from Fe II than from Fe I lines. To place this systematic effect in perspective, we present in Table 2 the customary table showing the effect on the derived Fe abundance from Fe I and Fe II lines of small changes to the model atmosphere parameters. By inspection, we see that a combination of changes to the parameters within their measurement uncertainties would allow the neutral and ionized lines to return the same Fe abundance for the coolest giants. For example, a temperature correction running from an increase of $T_{\text {eff }}$ by $100 \mathrm{~K}$ at $3900 \mathrm{~K}$ and vanishing at about $4200 \mathrm{~K}$ would remove the discrepancy among the coolest stars and ensure that all stars gave the same
Fe abundance. This mild revision of the $T_{\text {eff }}$ scale seems preferable to the supposition that the discrepancy reflects the appearance of non-LTE (or other) effects in the atmospheres of the coolest stars. If the Fe abundance is derived from Fe I and Fe II lines of stars with $T_{\text {eff }}>4200 \mathrm{~K}$, the weighted mean abundance is $\log \epsilon(\mathrm{Fe})=5.88 \pm 0.01(\sigma=0.03)$. Taking the Kurucz et al. (1984) solar atlas, we measured the equivalent widths of Fe I and Fe II lines (same set of lines as measured in the giants). The measured equivalent widths range from 10 to $90 \mathrm{m \AA}$. Using a Kurucz model with $T_{\text {eff }}=5770 \mathrm{~K}, \log g=4.44, \xi_{\mathrm{t}}=$ $1.0 \mathrm{~km} \mathrm{~s}^{-1}$, we derive $\log \epsilon(\mathrm{Fe} \mathrm{I})=7.51$ and $\log \epsilon(\mathrm{Fe}$ II $)=7.50$ using the van der Waals line damping parameter (Unsöld approximation multiplied by a factor recommended by the Blackwell group). Using the Holweger \& Müller (1974) empirical model atmosphere with $\xi_{\mathrm{t}}=1.15 \mathrm{~km} \mathrm{~s}^{-1}$, we derive $\log \epsilon(\mathrm{Fe} \mathrm{I})=7.53$ and $\log \epsilon(\mathrm{Fe}$ II $)=7.47$. Adopting a solar metallicity of $\log \epsilon(\mathrm{Fe})=7.50$, we obtain $[\mathrm{Fe} / \mathrm{H}]=-1.62$ for NGC 6752 with no evidence for a star-to-star variation.

Our cluster metallicity $[\mathrm{Fe} / \mathrm{H}]=-1.62$ is similar to previously published values. Grundahl et al. (2002, private communication) also obtained $[\mathrm{Fe} / \mathrm{H}]=-1.62$ from highresolution spectra of their red giants. Gratton et al. (2001) report $[\mathrm{Fe} / \mathrm{H}]=-1.42$ from UVES spectra of turn-off stars and subgiants. Kraft \& Ivans (2003) find $[\mathrm{Fe} / \mathrm{H}]_{\mathrm{I}}=-1.50$ and $[\mathrm{Fe} / \mathrm{H}]_{\mathrm{II}}=-1.42$ using Kurucz models and $[\mathrm{Fe} / \mathrm{H}]_{\mathrm{I}}=-1.51$ and $[\mathrm{Fe} / \mathrm{H}]_{\mathrm{II}}=-1.50$ using MARCS models. Other analyses 
Table 2. Abundance dependences on model parameters.

\begin{tabular}{lcccccc}
\hline \hline Abundance & $\Delta T_{\text {eff }} \pm 50 \mathrm{~K}$ & $\begin{array}{c}\Delta \log g \pm 0.2 \\
\text { NGC 6752-mg0 }\end{array}$ & $\Delta \xi_{\mathrm{t}} \pm 0.2$ & $\Delta T_{\text {eff }} \pm 50 \mathrm{~K}$ & $\Delta \log g \pm 0.2$ & $\Delta \xi_{\mathrm{t}} \pm 0.2$ \\
& & & \multicolumn{3}{c}{ NGC 6752-mg24 $^{b}$} \\
\hline $\log \epsilon(\mathrm{Fe} \mathrm{I})$ & \pm 0.02 & $\mp 0.01$ & $\mp 0.02$ & \pm 0.06 & $\mp 0.01$ & $\mp 0.02$ \\
$\log \epsilon(\mathrm{Fe} \mathrm{II})$ & $\mp 0.09$ & \pm 0.04 & $\mp 0.03$ & $\mp 0.01$ & \pm 0.07 & $\mp 0.03$ \\
$\log \epsilon(\mathrm{Fe})$ & \pm 0.00 & $\mp 0.01$ & $\mp 0.03$ & \pm 0.04 & \pm 0.01 & $\mp 0.02$ \\
$\log \epsilon(\mathrm{O})$ & $\mp 0.01$ & \pm 0.04 & $\mp 0.01$ & \pm 0.02 & \pm 0.08 & \pm 0.00 \\
$\log \epsilon(\mathrm{Na})$ & \pm 0.05 & $\mp 0.03$ & $\mp 0.02$ & \pm 0.05 & $\mp 0.01$ & \pm 0.00 \\
$\log \epsilon(\mathrm{Mg})$ & \pm 0.03 & $\mp 0.03$ & $\mp 0.06$ & \pm 0.06 & $\mp 0.02$ & $\mp 0.04$ \\
$\log \epsilon(\mathrm{Al})$ & \pm 0.05 & $\mp 0.02$ & $\mp 0.02$ & \pm 0.04 & $\mp 0.01$ & $\mp 0.01$ \\
\hline
\end{tabular}

${ }^{a} \operatorname{mg} 0: T_{\text {eff }}=3928 \mathrm{~K}, \log g=0.26 \mathrm{~cm} \mathrm{~s}^{-2}, \xi_{\mathrm{t}}=2.7 \mathrm{~km} \mathrm{~s}^{-1}$.

${ }^{b} \mathrm{mg} 24: T_{\text {eff }}=4511 \mathrm{~K}, \log g=1.31 \mathrm{~cm} \mathrm{~s}^{-2}, \xi_{\mathrm{t}}=1.9 \mathrm{~km} \mathrm{~s}^{-1}$.

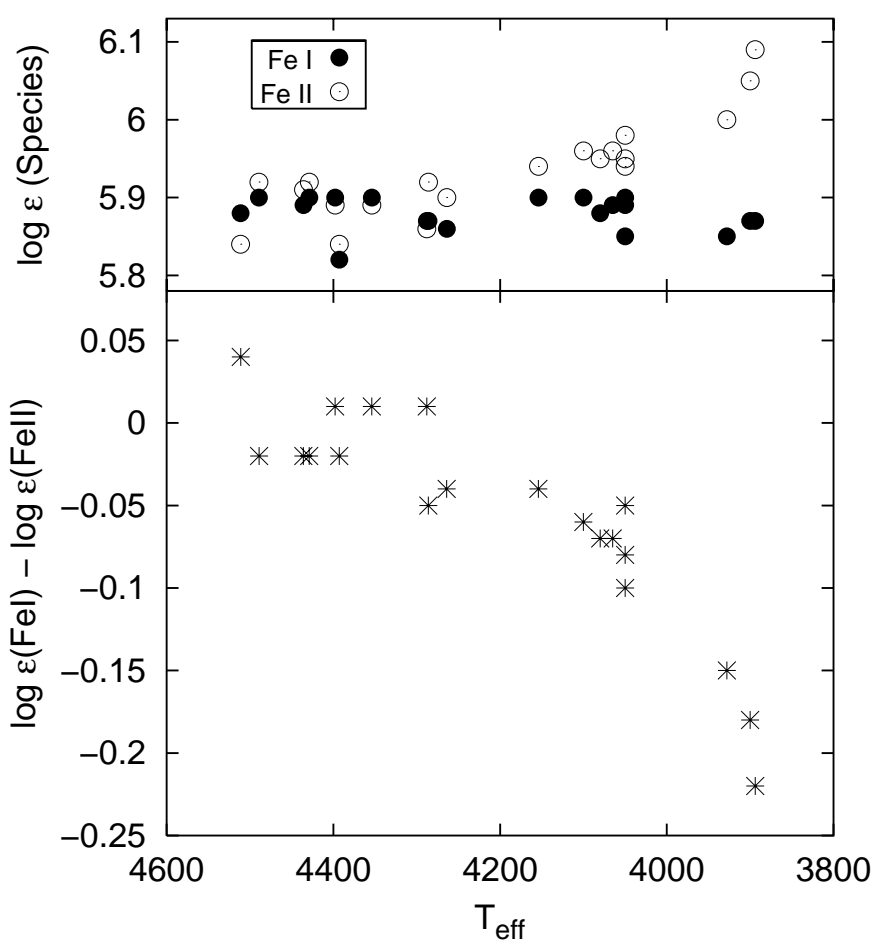

Fig. 3. In the upper panel we plot $\log \epsilon(\mathrm{Fe}$ ) (filled circles) and $\log \epsilon$ (FeII) (open circles) versus $T_{\text {eff }}$. In the lower panel we plot the difference between Fe I and Fe II abundance against $T_{\text {eff }}$. We find that $\log \epsilon(\mathrm{FeI})$ remains essentially constant as $T_{\text {eff }}$ changes while $\log \epsilon \mathrm{Fe}(\mathrm{II})$ increases with decreasing $T_{\text {eff }}$ beginning around $T_{\text {eff }}=$ $4200 \mathrm{~K}$.

of NGC 6752's giants include $[\mathrm{Fe} / \mathrm{H}]=-1.42$ (Carretta \& Gratton 1997), -1.58 (Minniti et al. 1993), and -1.54 (Zinn \& West 1984). That we find NGC 6752 to be slightly more metal-poor than other investigators is not relevant to our main purpose which is the measurement of the $\mathrm{Mg}$ isotopic ratios and the abundances of the light elements $\mathrm{O}, \mathrm{Na}, \mathrm{Mg}$, and $\mathrm{Al}$.

For the three additional stars, the VLT reobservations revealed no discernable systematic differences in derived abundances when compared to the original observations and analysis (Shetrone 1998).

\section{Oxygen, sodium, magnesium and aluminium abundances}

Red giants in NGC 6752 are known to show star-to-star variations in the $\mathrm{C}$ and $\mathrm{N}$ abundances. Norris et al. (1981) found the variations in $\mathrm{CN}$ band strength anticorrelated with $\mathrm{CH}$ band strength. Suntzeff \& Smith (1991), as noted earlier, found the star-to-star variations to extend to main sequence stars. Extension of star-to-star abundance variations to $\mathrm{O}, \mathrm{Na}, \mathrm{Mg}$, and $\mathrm{Al}$ has been reported for several clusters (e.g. Kraft et al. 1997; Sneden et al. 1997; Ivans et al. 2001). In the case of NGC 6752, this extension has been made for a handful of bright giants (Norris \& Da Costa 1995; Minniti et al. 1996), red giant branch stars (Grundahl et al. 2002), and turn-off stars and early subgiants (Gratton et al. 2001). Here, we extend the search for abundance variations to a large sample of stars at the top of the red giant branch.

For $\mathrm{O}, \mathrm{Na}, \mathrm{Mg}$, and $\mathrm{Al}$, abundances were derived from the measured equivalent widths using the $g f$-values from Shetrone (1996a) and from a list compiled by Luck (1993, private communication). The radial velocity of the cluster stars ranged from -17 to $-46 \mathrm{~km} \mathrm{~s}^{-1}$ allowing easy removal of night sky emission without affecting stellar $\mathrm{O}$ absorption lines. Our line list is presented in Table 3. The elemental abundances are presented in Table 1 and the anticorrelations between $\mathrm{O}-\mathrm{Na}$ and $\mathrm{Mg}-\mathrm{Al}$ can be seen in Fig. 4. As seen in previous studies, we find an anticorrelation between oxygen and sodium where stars with low abundances of $\mathrm{O}$ have correspondingly large abundances of $\mathrm{Na}$. Within the bright giants, oxygen varies by $\Delta \log \epsilon(\mathrm{O})=0.6$, sodium varies by $\Delta \log \epsilon(\mathrm{Na})=0.8$, aluminium varies by $\Delta \log \epsilon(\mathrm{Al})=1.1$ while magnesium varies by $\Delta \log \epsilon(\mathrm{Mg})=0.2$. There is a weak anticorrelation between $\mathrm{Mg}$ and $\mathrm{Al}$.

Taking NGC 6752-mg0 and NGC 6752-mg24, stars at the cool and warm end of our sample, we determined the dependence of the derived abundances upon the model parameters (see Table 2). Our results show that oxygen decreases by about $0.6 \mathrm{dex}$ as sodium increases by about $0.8 \mathrm{dex}$ (Fig. 4). These changes in elemental abundance are far greater than the estimated uncertainties in Table 2. The aluminium abundance range is about 1.1 dex, again at least an order of magnitude larger than the estimated uncertainty. The small spread of the $\mathrm{Mg}$ abundance across our sample probably exceeds the 
Table 3. Atomic line list.

\begin{tabular}{lccclccc}
\hline \hline Species & Wavelength $(\AA)$ & $E P(\mathrm{eV})$ & $\log g f$ & Species & Wavelength $(\AA)$ & $E P(\mathrm{eV})$ & $\log g f$ \\
\hline $\mathrm{O}$ I & 6300.30 & 0.00 & -9.75 & $\mathrm{Fe}$ I & 5321.11 & 4.43 & -1.19 \\
$\mathrm{O}$ I & 6363.78 & 0.02 & -10.25 & $\mathrm{Fe}$ I & 5322.04 & 2.28 & -3.03 \\
$\mathrm{Na}$ I & 4982.83 & 2.10 & -0.91 & $\mathrm{Fe}$ I & 5373.71 & 4.47 & -0.71 \\
$\mathrm{Na}$ I & 5682.65 & 2.10 & -0.71 & $\mathrm{Fe}$ I & 5466.40 & 4.37 & -0.57 \\
$\mathrm{Na}$ I & 5688.22 & 2.10 & -0.40 & $\mathrm{Fe}$ I & 5487.75 & 4.32 & -0.65 \\
$\mathrm{Na}$ I & 6154.23 & 2.10 & -1.56 & $\mathrm{Fe}$ I & 5522.45 & 4.21 & -1.40 \\
$\mathrm{Na}$ I & 6160.75 & 2.10 & -1.26 & $\mathrm{Fe}$ I & 5554.90 & 4.55 & -0.38 \\
$\mathrm{Mg}$ I & 5528.40 & 4.35 & -0.36 & $\mathrm{Fe}$ I & 5560.21 & 4.43 & -1.04 \\
$\mathrm{Mg}$ I & 5711.09 & 4.35 & -1.73 & $\mathrm{Fe}$ I & 5567.39 & 2.61 & -2.80 \\
$\mathrm{Mg}$ I & 6318.71 & 5.11 & -1.97 & $\mathrm{Fe}$ I & 5584.77 & 3.57 & -2.17 \\
$\mathrm{Mg}$ I & 6319.24 & 5.11 & -2.20 & $\mathrm{Fe}$ I & 5618.63 & 4.21 & -1.26 \\
$\mathrm{Al}$ I & 5557.07 & 3.14 & -1.95 & $\mathrm{Fe}$ I & 5624.02 & 4.39 & -1.33 \\
$\mathrm{Al}$ I & 6696.02 & 3.14 & -1.57 & $\mathrm{Fe}$ I & 5633.95 & 4.99 & -0.12 \\
$\mathrm{Al}$ I & 6698.67 & 3.14 & -1.89 & $\mathrm{Fe}$ I & 5635.82 & 4.26 & -1.74 \\
$\mathrm{Fe}$ I & 4817.78 & 2.22 & -3.53 & $\mathrm{Fe}$ I & 5638.26 & 4.22 & -0.72 \\
$\mathrm{Fe}$ I & 4848.88 & 2.28 & -3.40 & $\mathrm{Fe}$ I & 5679.02 & 4.65 & -0.77 \\
$\mathrm{Fe}$ I & 4877.61 & 3.00 & -3.15 & $\mathrm{Fe}$ I & 5705.47 & 4.30 & -1.36 \\
$\mathrm{Fe}$ I & 4896.44 & 3.88 & -1.90 & $\mathrm{Fe}$ I & 5731.76 & 4.26 & -1.15 \\
$\mathrm{Fe}$ I & 4930.32 & 3.96 & -1.20 & $\mathrm{Fe}$ II & 4993.35 & 2.81 & -3.67 \\
$\mathrm{Fe}$ I & 4969.92 & 4.22 & -0.75 & $\mathrm{Fe}$ II & 5197.56 & 3.23 & -2.25 \\
$\mathrm{Fe}$ I & 5002.79 & 3.40 & -1.44 & $\mathrm{Fe}$ II & 5234.62 & 3.22 & -2.24 \\
$\mathrm{Fe}$ I & 5029.62 & 3.42 & -1.90 & $\mathrm{Fe}$ II & 5264.81 & 3.23 & -3.19 \\
$\mathrm{Fe}$ I & 5090.77 & 4.26 & -0.36 & $\mathrm{Fe}$ II & 5284.10 & 2.89 & -3.01 \\
$\mathrm{Fe}$ I & 5121.64 & 4.28 & -0.72 & $\mathrm{Fe}$ II & 5414.05 & 3.22 & -3.62 \\
$\mathrm{Fe}$ I & 5143.72 & 2.20 & -3.79 & $\mathrm{Fe}$ II & 5425.25 & 3.20 & -3.21 \\
$\mathrm{Fe}$ I & 5222.39 & 2.28 & -3.68 & $\mathrm{Fe}$ II & 5534.83 & 3.25 & -2.77 \\
$\mathrm{Fe}$ I & 5288.53 & 3.69 & -1.51 & & & & \\
\hline
\end{tabular}

estimated uncertainties; note the sensitivity of the $\mathrm{Mg}$ abundance to the microturbulence (Table 2). The Na and Al abundances are well correlated.

In order to compare our light element abundances with those for less luminous red giants, we have reanalyzed Grundahl et al.'s $W_{\lambda}$ s for lines in our line list using Kurucz models for the atmospheric parameters found by them. Results plotted in Fig. 4 show excellent agreement for $\mathrm{O}, \mathrm{Na}, \mathrm{Mg}$, and $\mathrm{Al}$ abundances with respect to absolute abundance and total spread. Grundahl et al.'s sample appears to extend the $\mathrm{Na}-\mathrm{O}$ anticorrelation at the high-Na low-O end. If we use the MARCS model atmospheres used by Grundahl et al., their abundances are typically about 0.15 dex lower. Regarding the $\mathrm{Mg}-\mathrm{Al}$ anticorrelation, we note that for both our sample and the Grundahl et al. sample, the total $\log \epsilon(\mathrm{Mg})+\log \epsilon(\mathrm{Al})$ is constant. This is consistent with previous work by Shetrone (1996a, 1998) for M 13 and NGC 6752.

A comparison is possible with results for turn-off stars and subgiants using $[\mathrm{O} / \mathrm{Fe}],[\mathrm{Na} / \mathrm{Fe}],[\mathrm{Mg} / \mathrm{Fe}]$, and $[\mathrm{Al} / \mathrm{Fe}]$ published by Gratton et al. (2001). Their $\mathrm{O}$ abundances are derived from the 7771-7774 $\AA$ triplet; non-LTE effects were considered but are 0.1 dex or smaller. The $\mathrm{Na}$ abundance is based on the "quite strong" $8183 \AA$ and $8194 \AA$ doublet and includes a small non-LTE correction. Converting published results to abundances $\log \epsilon$ (Species), we find that their Na-O anticorrelation parallels ours ${ }^{2}$. Offsets to Gratton et al.'s abundances of

\footnotetext{
${ }^{2}$ In this conversion, we assume solar abundances of 6.33 for $\mathrm{Na}$ and 8.92 for $\mathrm{O}$, and $[\mathrm{Fe} / \mathrm{H}]=-1.42$, Gratton et al.'s value.
}

-0.2 dex for $\mathrm{Na}$ and -0.2 dex for $\mathrm{O}$ superimpose their results on ours (see Fig. 4).

Gratton et al.'s Al abundances for subgiants are derived from $8774 \AA$ doublet and include a small non-LTE correction. For the turn-off stars, the Al abundance depends on the $3944 \AA$ and $3961 \AA$ resonance lines and a large non-LTE correction. The $\mathrm{Mg}$ abundance was found from a set of lines in the blue and yellow regions of the spectrum that likely includes lines from our line list. The range in $\mathrm{Al}$ abundances ${ }^{3}$ from Gratton et al.'s sample runs from about 4.6 to 5.8 , which is displaced to lower abundances by about 0.4 dex relative to our range. Gratton et al.'s Mg abundances are systematically lower than ours. Their result $[\mathrm{Mg} / \mathrm{Fe}] \simeq 0.0$ contrasts with $[\mathrm{Mg} / \mathrm{Fe}] \simeq 0.6$ for our and Grundahl et al.'s samples of giants. Offsets to Gratton et al.'s abundances of +0.2 dex for $\mathrm{Mg}^{4}$ and +0.4 dex for Al superimpose their results on ours (see Fig. 4). Clearly, it would be helpful to understand the origins of the differences in the $\mathrm{Mg}$ and $\mathrm{Al}$ abundances. In particular, interpretations of star-to-star abundance variations are severely constrained if the differences are real and not a reflection of systematic errors in one or both of the abundance analyses.

Our stellar sample includes two field stars analyzed by Shetrone (1996b). The O, Na, Mg, and Al abundances obtained from our $W_{\lambda} \mathrm{s}$ and a Kurucz model for Shetrone's

\footnotetext{
${ }^{3}$ Conversion of $[\mathrm{Al} / \mathrm{Fe}]$ to $\mathrm{Al}$ abundance was made assuming a solar $\mathrm{Al}$ abundance of 6.47 and $[\mathrm{Fe} / \mathrm{H}]=-1.42$.

${ }^{4}$ Conversion of $[\mathrm{Mg} / \mathrm{Fe}]$ to $\mathrm{Mg}$ abundance was made assuming a solar $\mathrm{Mg}$ abundance of 7.58 and $[\mathrm{Fe} / \mathrm{H}]=-1.42$.
} 

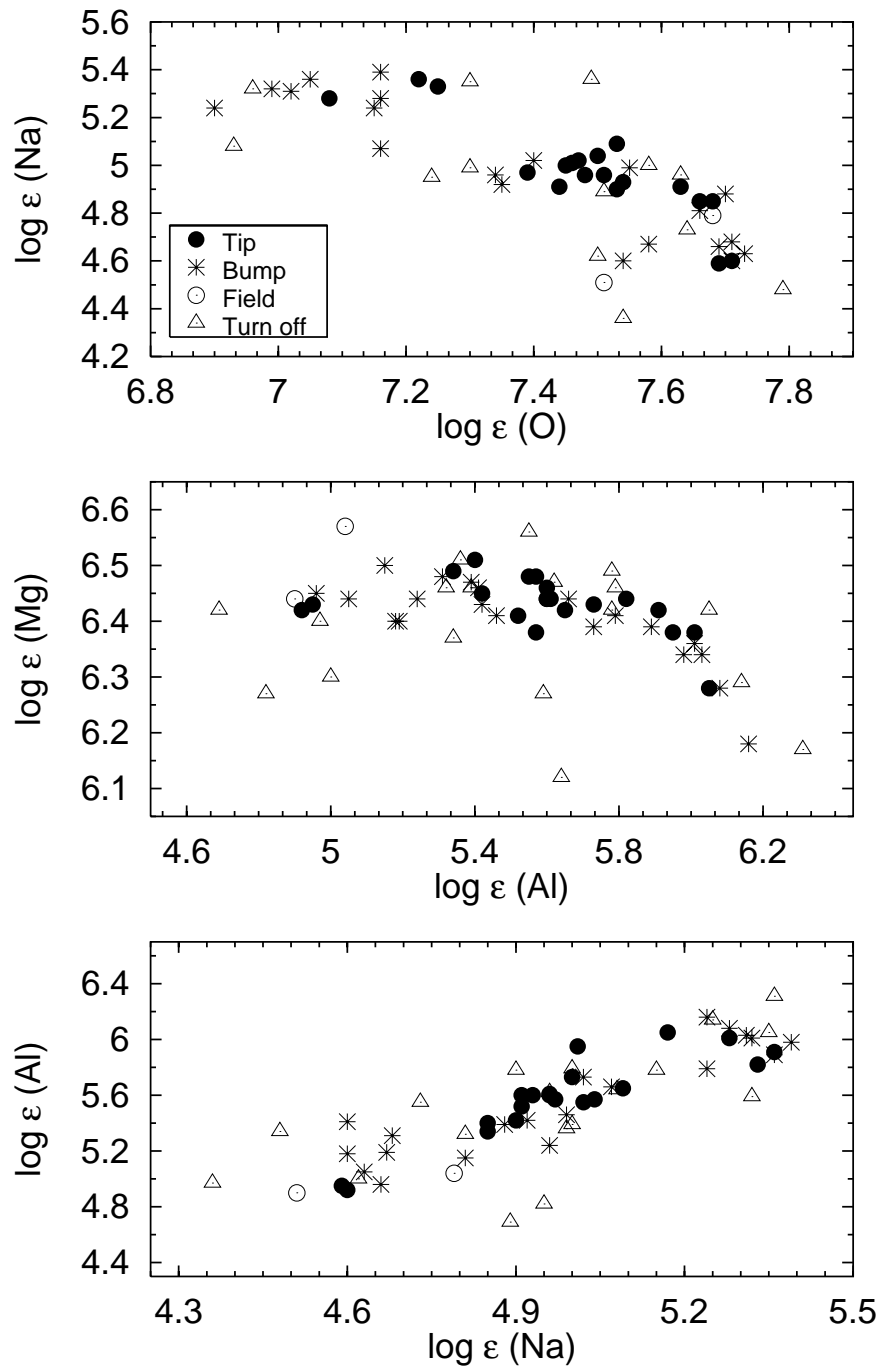

Fig. 4. Observed anti-correlations between $\mathrm{O}$ and $\mathrm{Na}$ (upper), $\mathrm{Al}$ and $\mathrm{Mg}$ (middle), and the correlation between $\mathrm{Na}$ and $\mathrm{Al}$ (lower). The RGB tip stars from this study (closed circles), the Grundahl et al. (2002) RGB bump stars (asterisks), the Gratton et al. (2001) turnoff stars and early subgiants (open triangles), and the 2 field giants (open circles) are plotted. Abundances from Gratton et al. (2001) were shifted onto our scale (see text).

chosen $T_{\text {eff }}$ and $\log g$ are given in Table 1. We add these stars to Fig. 4 by adjusting the abundances for the small metallicity differences between the stars and NGC 6752 by supposing that $\Delta \log \epsilon($ Species $)=\Delta \log \epsilon(\mathrm{Fe})$. It is to be noted that the comparison stars fall at the high $\mathrm{O}$, low $\mathrm{Na}$, high $\mathrm{Mg}$, and low $\mathrm{Al}$ end of the distribution defined by the red giants. Our Fe, O, Na, $\mathrm{Mg}$, and $\mathrm{Al}$ abundances agree with Shetrone's values to better than 0.1 dex with just two minor exceptions: our $\mathrm{O}$ abundance for $\mathrm{HD} 141531$ is 0.14 dex larger and the $\mathrm{Al}$ abundance for HD 103036 is 0.2 dex larger than Shetrone's values.

\section{Magnesium isotopic abundances}

The A-X band of $\mathrm{MgH}$ is present in the spectra of NGC 6752's red giants. Shetrone's pioneering study of $\mathrm{Mg}$ isotopic abundances in red giants from M13, a cluster of similar metallicity with similarly pronounced $\mathrm{O}, \mathrm{Na}, \mathrm{Mg}$, and $\mathrm{Al}$ abundance

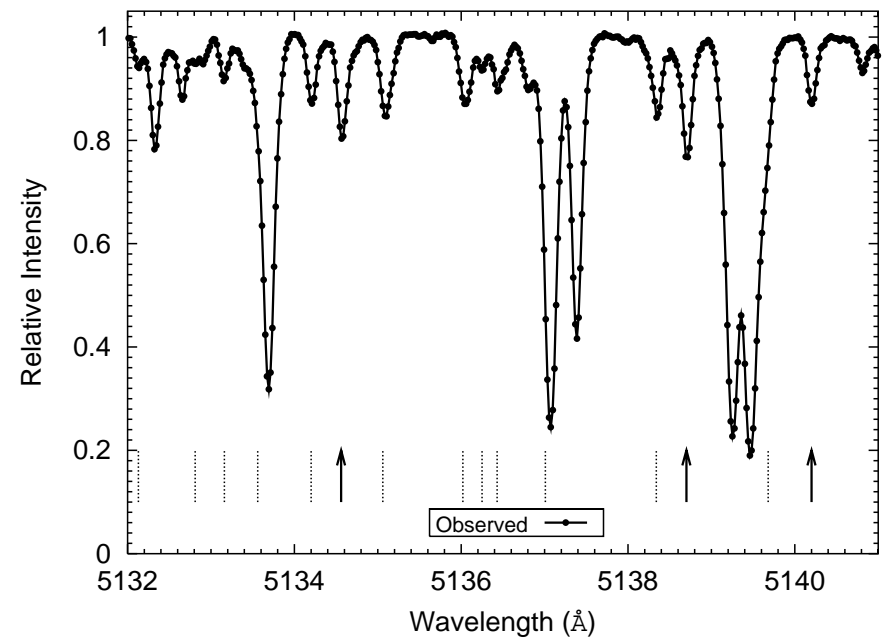

Fig. 5. Spectra of NGC 6752-mg6 from 5132 to $5141 \AA$ A. The positions of the $\mathrm{MgH}$ A-X $0-0$ and $\mathrm{MgH}$ A-X 1-1 lines are marked below the spectrum. The majority of $\mathrm{MgH}$ lines are unsuitable for isotopic analysis due to blends. The positions of the 3 features that we used to derive the isotopic ratios are highlighted with arrows.

variations, demonstrated the feasibility of the program to determine the isotopic abundances. Shetrone (1998) also determined $\mathrm{Mg}$ isotopic ratios for 4 bright giants in NGC 6752. Examination of the spectra confirms that $\mathrm{MgH}$ lines are present - see Fig. 5. More importantly, a mere glance at the spectra shows that there is a star-to-star variation in the isotopic abundance ratios. This is well shown by Fig. 6 where we overplot the spectra of the three coolest (brightest) giants in the regions providing three of the key $\mathrm{MgH}$ lines selected by McWilliam \& Lambert (1988) as indicators of the isotopic abundance ratios. Note the asymmetric profiles of the $\mathrm{MgH}$ lines with the ${ }^{25} \mathrm{MgH}$ and ${ }^{26} \mathrm{MgH}$ lines providing the trailing red wing. For each of the three lines (and others), the relative strengths of the asymmetry are the same with the strongest asymmetry occurring for $\mathrm{mg} 0$ and the weakest for mg2. Inspection of Fig. 6 also shows that the differences in the profile occur at the wavelength of the ${ }^{26} \mathrm{MgH}$ components but appear to vanish at the wavelength of the ${ }^{25} \mathrm{MgH}$ components; the abundance differences are largely in the ${ }^{26} \mathrm{Mg} /{ }^{24} \mathrm{Mg}$ ratio not in the ${ }^{25} \mathrm{Mg} /{ }^{24} \mathrm{Mg}$ ratio. To convert these impressions to quantitative estimates, spectrum syntheses were generated and fitted to the observed spectra.

In the quantitative analysis, three $\mathrm{MgH}$ features recommended by McWilliam \& Lambert (1988) and used by Gay \& Lambert (2000) were analyzed. The first line at $5134.6 \AA$ is a blend of the $Q_{1}(23)$ and $R_{2}(11)$ lines from the $0-0$ band. There are 2 similar features on either side of this line where contributions from other lines render them unsuitable for the extraction of reliable isotopic ratios (Tomkin \& Lambert 1980). The second line at $5138.7 \AA$ is a blend of the $0-0 Q_{1}(22)$ and 1-1 $Q_{2}$ (14) lines in the wing of a strong atomic line. The last line employed in the determination of the isotopic ratios is at $5140.2 \AA$, a blend of the $0-0 R_{1}(10)$ and $1-1 R_{2}(4)$ lines. The macroturbulence was determined with a Gaussian representing the combined effect of the atmospheric turbulence, stellar rotation, and the instrumental profile. This Gaussian was fixed by fitting the profiles of unblended lines namely the Ni I 5115.4 


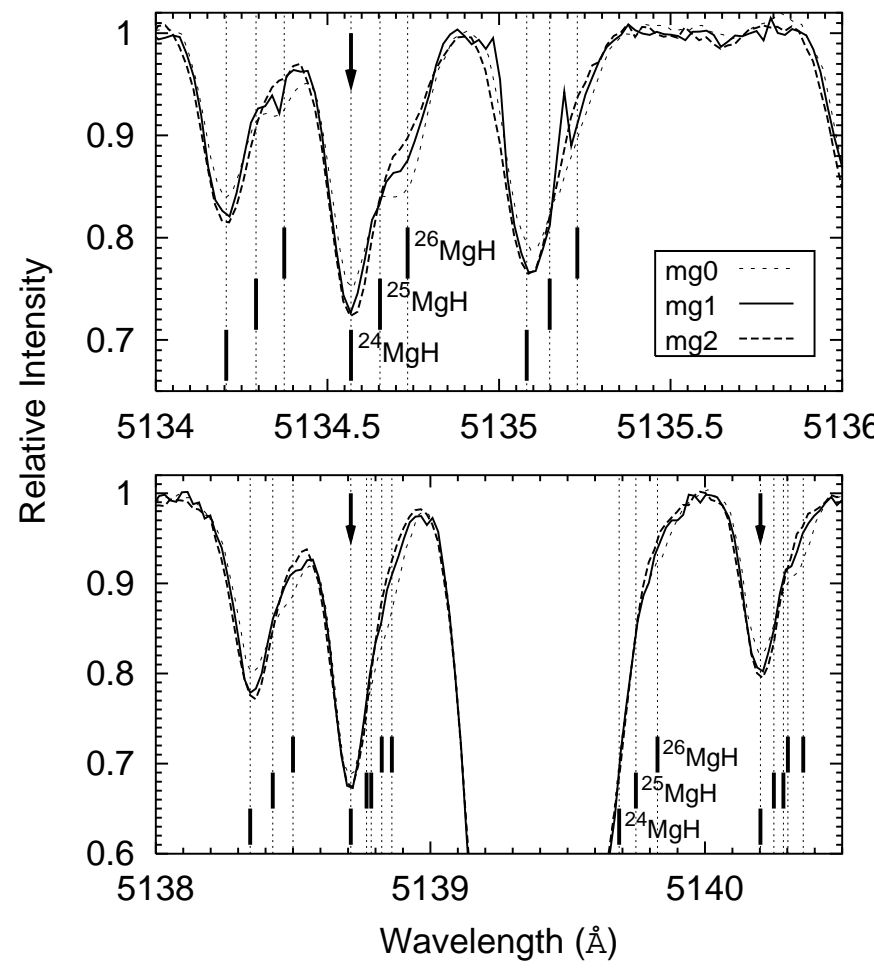

Fig. 6. Spectra of NGC 6752-mg0, mg1, and mg2 from 5134 to $5136 \AA$ (upper) and from 5138 to $5140.5 \AA$ (lower). The positions of the ${ }^{24} \mathrm{MgH},{ }^{25} \mathrm{MgH}$, and ${ }^{26} \mathrm{MgH}$ lines are shown. The lines used to derive the $\mathrm{Mg}$ isotopic ratios are identified by arrows. Striking differences in the line profiles are immediately obvious where ${ }^{26} \mathrm{MgH}$ appears to be varying between the 3 stars.

and the Ti I $5145.5 \AA$. In all stars, these lines were slightly stronger than the recommended $\mathrm{MgH}$ lines. The line list was identical to that used in Gay \& Lambert (2000) and included the following elements $\mathrm{C}, \mathrm{Mg}, \mathrm{Sc}, \mathrm{Ti}, \mathrm{Cr}, \mathrm{Fe}, \mathrm{Co}, \mathrm{Ni}$, and $\mathrm{Y}$. The ${ }^{25} \mathrm{Mg}$ and ${ }^{26} \mathrm{Mg}$ abundances were adjusted by trial and error until the profiles of the 3 recommended features were best fitted where we treated each of the 3 regions independently. The best fit was determined by eye (see Table 4 for Mg isotopic ratios). Examples of spectrum syntheses are shown in Fig. 7. A point deserving of emphasis is that the red asymmetry of $\mathrm{MgH}$ lines demands major contributions from the ${ }^{25} \mathrm{Mg}$ and especially the ${ }^{26} \mathrm{Mg}$ isotopes. A spectrum computed for pure ${ }^{24} \mathrm{Mg}$ clearly does not fit the observed spectrum. We refrain from using the $\mathrm{MgH}$ features to determine the $\mathrm{Mg}$ abundance as slight variations in the adopted temperature result in large changes to the $\mathrm{Mg}$ abundance required to fit the molecular lines.

Next, we sought an unbiased method for determining the best fit to the data. Following the successful work by Nissen et al. $(1999,2000)$ on the $\mathrm{Li}$ isotopic ratios, we chose to use a $\chi^{2}$ analysis. Such an analysis has the benefits of being unbiased as well as allowing us to quantify the errors in the fits. Our free parameters to be varied were (1) ${ }^{25} \mathrm{Mg} /{ }^{24} \mathrm{Mg}$, (2) ${ }^{26} \mathrm{Mg} /{ }^{24} \mathrm{Mg}$, and (3) $\log \epsilon(\mathrm{Mg})$ where we treated each of the three recommended features independently. This choice of free parameters ensured that equal step sizes produced comparable changes in the synthetic spectra. Our initial guesses for the isotopic ratios were the optimum values found using the traditional

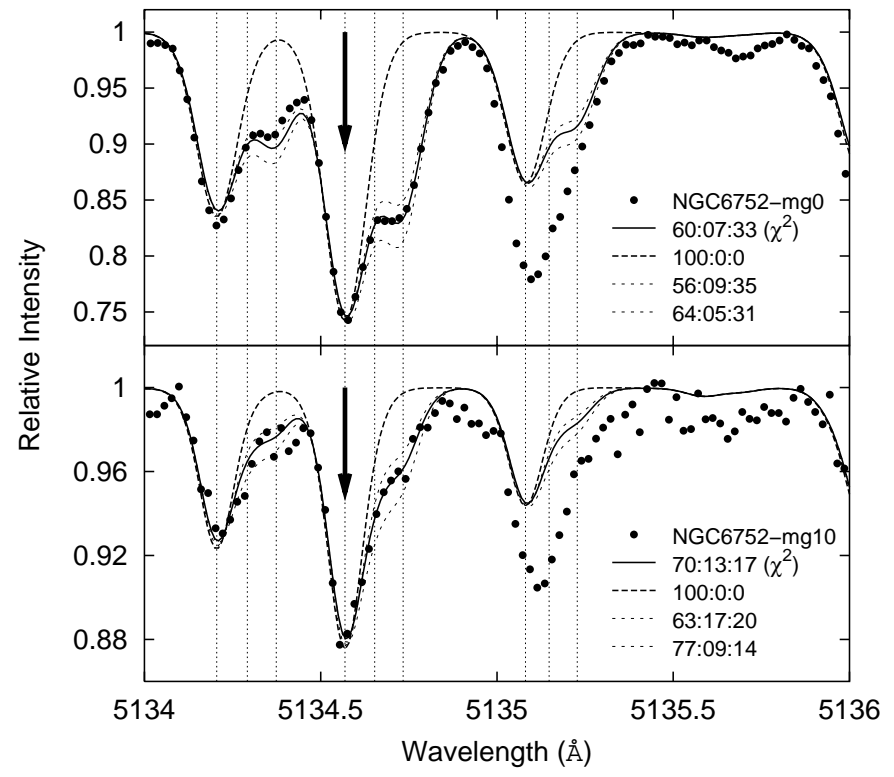

Fig. 7. Spectra of NGC 6752-mg0 (upper) and NGC 6752-mg10 (lower) from 5134.0 to $5136.0 \AA$. The feature we are interested in fitting is highlighted by the arrow. The positions of the ${ }^{24} \mathrm{MgH},{ }^{25} \mathrm{MgH}$, and ${ }^{26} \mathrm{MgH}$ lines are indicated by dashed lines. The closed circles represent the observed spectra. The synthetic spectrum generated using the isotopic ratios determined by $\chi^{2}$ analysis is given by the solid line: the ${ }^{24} \mathrm{Mg}:{ }^{25} \mathrm{Mg}:{ }^{26} \mathrm{Mg}$ ratios are given on the figure. Unsatisfactory ratios are plotted as dotted lines.

method. We explored a large parameter space around our initial guesses. The $\chi^{2}$ was calculated via $\chi^{2}=\Sigma\left(O_{i}-S_{i}\right)^{2} / \sigma^{2}$ where $O_{i}$ is the observed spectrum point, $S_{i}$ is the synthesis, and $\sigma=(S / N)^{-1}$. We determined the optimum values for ${ }^{25} \mathrm{Mg} /{ }^{24} \mathrm{Mg},{ }^{26} \mathrm{Mg} /{ }^{24} \mathrm{Mg}$, and $\log \epsilon(\mathrm{Mg})$ by finding the minima in $\chi^{2}$. We then searched a smaller range in parameter space with finer grid spacing centered upon the optimum values. We again measured $\chi^{2}$ locating the minima for ${ }^{25} \mathrm{Mg} /{ }^{24} \mathrm{Mg},{ }^{26} \mathrm{Mg} /{ }^{24} \mathrm{Mg}$, and $\log \epsilon(\mathrm{Mg})$ in each region, in each star. Therefore, for a given star, we obtained 3 optimal values for ${ }^{25} \mathrm{Mg} /{ }^{24} \mathrm{Mg}$ and ${ }^{26} \mathrm{Mg} /{ }^{24} \mathrm{Mg}$ from which we readily recovered the ratio ${ }^{24} \mathrm{Mg}:{ }^{25} \mathrm{Mg}:{ }^{26} \mathrm{Mg}$ (see Table 4 and Figs. 7 and 8). In finding the optimal values we generated and tested over 1500 synthetic spectra per region. The minimum $\chi_{\text {red }}^{2}=\chi^{2} / v$, where $v$ is the number of degrees of freedom in the fit, was sufficiently close to 1 . However, since we were interested in a single isotopic ratio for a given star, first we needed to quantify the errors in the ratios ${ }^{25} \mathrm{Mg} /{ }^{24} \mathrm{Mg}$ and ${ }^{26} \mathrm{Mg} /{ }^{4} \mathrm{Mg}$ for each region. Following Bevington \& Robinson (1992) and Nissen et al. (1999, 2000), we plotted $\Delta \chi^{2}=\chi^{2}-\chi_{\min }^{2}$ against the ratios ${ }^{25} \mathrm{Mg} /{ }^{24} \mathrm{Mg}$ and ${ }^{26} \mathrm{Mg} /{ }^{24} \mathrm{Mg}$ (see Fig. 9). We took $\Delta \chi^{2}=1$ to be the $1 \sigma$ confidence limit for determining ${ }^{25} \mathrm{Mg} /{ }^{24} \mathrm{Mg}$ or ${ }^{26} \mathrm{Mg} /{ }^{4} \mathrm{Mg}$. Thus for each region of each star, we paired an uncertainty to the optimized value for ${ }^{25} \mathrm{Mg} /{ }^{24} \mathrm{Mg}$ or ${ }^{26} \mathrm{Mg} /{ }^{24} \mathrm{Mg}$. A weighted mean was calculated giving a single value of ${ }^{24} \mathrm{Mg}:{ }^{25} \mathrm{Mg}:{ }^{26} \mathrm{Mg}$ for each star (see Table 4).

We comment on the accuracy of the ${ }^{25} \mathrm{Mg} /{ }^{24} \mathrm{Mg}$ and ${ }^{26} \mathrm{Mg} /{ }^{24} \mathrm{Mg}$ ratios. As stated in Gay \& Lambert (2000), the ratio ${ }^{26} \mathrm{Mg} /{ }^{24} \mathrm{Mg}$ is more accurately determined than ${ }^{25} \mathrm{Mg} /{ }^{24} \mathrm{Mg}$ due to the larger isotopic shift. That is, ${ }^{26} \mathrm{MgH}$ is less blended with 
Table 4. Magnesium isotopic ratios for program stars $\left({ }^{24} \mathrm{Mg}:{ }^{25} \mathrm{Mg}:{ }^{26} \mathrm{Mg}\right)$.

\begin{tabular}{lccccccc}
\hline \hline Name & \multicolumn{3}{c}{ Initial guesses $^{a}$} & \multicolumn{3}{c}{ Optimum value $^{b}$} & Final ratio $^{c}$ \\
& Region 1 & Region 2 & Region 3 & Region 1 & Region 2 & Region 3 & \\
\hline NGC 6752-mg0 & $60: 08: 32$ & $61: 10: 29$ & $66: 08: 26$ & $60: 07: 33$ & $62: 08: 30$ & $67: 09: 24$ & $63: 08: 30$ \\
NGC 6752-mg1 & $67: 09: 24$ & $71: 09: 20$ & $76: 07: 17$ & $67: 08: 26$ & $71: 09: 20$ & $78: 07: 15$ & $71: 08: 21$ \\
NGC 6752-mg2 & $77: 05: 18$ & $79: 07: 14$ & $85: 05: 10$ & $76: 04: 20$ & $82: 06: 11$ & $90: 00: 10$ & $83: 03: 15$ \\
NGC 6752-mg3 & $75: 08: 17$ & $75: 10: 15$ & $80: 08: 12$ & $75: 08: 18$ & $76: 10: 14$ & $80: 09: 12$ & $76: 09: 15$ \\
NGC 6752-mg4 & $73: 08: 19$ & $73: 09: 18$ & $80: 06: 14$ & $72: 06: 22$ & $74: 07: 19$ & $80: 06: 15$ & $74: 06: 19$ \\
NGC 6752-mg5 & $75: 10: 15$ & $75: 10: 15$ & $81: 08: 11$ & $75: 09: 17$ & $75: 10: 16$ & $82: 06: 13$ & $76: 08: 16$ \\
NGC 6752-mg6 & $82: 09: 09$ & $83: 10: 07$ & $88: 08: 04$ & $83: 08: 10$ & $84: 09: 07$ & $86: 08: 05$ & $84: 08: 08$ \\
NGC 6752-mg8 & $70: 20: 10$ & $71: 17: 12$ & $76: 18: 06$ & $68: 18: 14$ & $68: 20: 12$ & $71: 21: 09$ & $68: 19: 12$ \\
NGC 6752-mg9 & $78: 14: 08$ & $76: 14: 10$ & Poor $S / N^{d}$ & $72: 13: 15$ & $74: 16: 09$ & $68: 24: 09$ & $72: 17: 10$ \\
NGC 6752-mg10 & $72: 15: 13$ & $70: 15: 15$ & $73: 13: 14$ & $70: 13: 17$ & $67: 10: 23$ & $73: 10: 17$ & $69: 11: 19$ \\
NGC 6752-mg12 & $88: 09: 03$ & $83: 11: 06$ & $83: 14: 03$ & $86: 08: 06$ & $82: 10: 08$ & $78: 15: 07$ & $83: 10: 07$ \\
NGC 6752-mg15 & $78: 07: 15$ & $76: 13: 11$ & Poor $S / N$ & $75: 13: 11$ & $76: 04: 20$ & $83: 00: 17$ & $77: 07: 16$ \\
NGC 6752-mg18 & $81: 10: 09$ & $78: 12: 10$ & Poor $S / N$ & $76: 10: 14$ & $76: 16: 08$ & $77: 10: 12$ & $77: 12: 12$ \\
NGC 6752-mg21 & $60: 00: 40$ & $60: 00: 40$ & Poor $S / N$ & $64: 01: 35$ & $68: 00: 32$ & $\ldots$ & $67: 00: 33^{e}$ \\
NGC 6752-mg22 & $60: 00: 40$ & $60: 00: 40$ & Poor $S / N$ & $62: 12: 26$ & $68: 05: 27$ & $\ldots$ & $65: 09: 26^{e}$ \\
NGC 6752-mg24 & $85: 10: 05$ & $85: 10: 05$ & Poor $S / N$ & $82: 08: 11$ & $85: 02: 14$ & $79: 22: 00$ & $82: 08: 10$ \\
NGC 6752-mg25 & $85: 10: 05$ & $85: 10: 05$ & Poor $S / N$ & $81: 06: 13$ & $85: 00: 15$ & $86: 00: 13$ & $84: 02: 14$ \\
NGC 6752-702 & $\ldots$ & $\ldots$ & $\ldots$ & $53: 08: 39$ & $51: 03: 46$ & $54: 18: 29$ & $53: 09: 39$ \\
NGC 6752-708 & $\ldots$ & $\ldots$ & $\ldots$ & $74: 16: 10$ & $80: 08: 12$ & $86: 04: 10$ & $78: 11: 11$ \\
NGC 6752-A88 & $\ldots$ & $\ldots$ & $\ldots$ & $71: 06: 23$ & $68: 15: 17$ & $71: 19: 10$ & $70: 11: 19$ \\
HD 103036 & $80: 05: 15$ & $80: 05: 15$ & $92: 03: 08$ & $90: 00: 10$ & $94: 00: 06$ & $100: 0: 0$ & $94: 00: 06$ \\
HD 141531 & $91: 04: 05$ & $94: 03: 03$ & $95: 03: 02$ & $88: 05: 07$ & $93: 00: 07$ & $97: 00: 03$ & $91: 02: 06$ \\
\hline
\end{tabular}

a Best fit determined by eye.

$b$ Best fit determined by $\chi^{2}$ analysis.

$c$ Weighted mean of the ratios derived for regions 1,2 , and 3 weighted by the $\chi^{2}$ errors. The formal statistical errors are dwarfed by the systematic uncertainties. We conservatively estimate errors in ${ }^{24} \mathrm{Mg}:{ }^{25} \mathrm{Mg}:{ }^{26} \mathrm{Mg}=(100-b-c): b: c$ as $b \pm 5$ and $c \pm 5$.

${ }^{d}$ When the $S / N$ was poor, we did not attempt to derive an isotopic ratio via the traditional method.

$e$ Region 3 was unusuable due to weakness of the lines and low signal-to-noise.

Note. - Region 1 is the line located at $5134.6 \AA$, Region 2 is the line located at $5138.7 \AA$, and Region 3 is located at $5140.2 \AA$.

the strong ${ }^{24} \mathrm{MgH}$ line than ${ }^{25} \mathrm{MgH}$. From Fig. 9, indeed we quantitatively verify that ${ }^{26} \mathrm{Mg} /{ }^{24} \mathrm{Mg}$ is more accurately determined than ${ }^{25} \mathrm{Mg} /{ }^{24} \mathrm{Mg}$. Also, we see that in general the ratios derived using Region 1 are more accurate than those derived using Regions 2 or 3. Region 1 has a tendency to give higher values of ${ }^{26} \mathrm{Mg} /{ }^{24} \mathrm{Mg}$ than those obtained from regions 2 or 3. The dwarfs analyzed by Gay \& Lambert (2000) did not show this trend. It is likely that these highly evolved giants are affected by unidentified lines which do not affect the dwarfs to the same degree. Table 4 suggests that the ratios derived using the traditional approach, where the eye determines the best fit, compare very favourably with ratios derived using the unbiased $\chi^{2}$ test. We now comment upon 2 stars which show unusual $\mathrm{MgH}$ lines, NGC 6752-mg21 and NGC 6752-mg22. Figures 10 and 11 highlight the remarkable asymmetries in the $\mathrm{MgH}$ lines. The atomic lines in these 2 stars are symmetric. Though the $\mathrm{MgH}$ lines are weak, our analyses suggest that both stars have ${ }^{26} \mathrm{Mg} / \mathrm{Mg} \geq 0.25$, a value comparable to NGC 6752-mg0 and NGC 6752-702.

We derived a mean isotopic ratio for each star based on the isotopic ratios of the 3 regions and their associated errors. The statistical uncertainties in the final ratios were also calculated, though we now comment on their relevance. Our formal statistical errors on the mean of the $\mathrm{Mg}$ isotopic ratios are small and fail to take into account systematic errors from various sources. Sources of uncertainties in the $\mathrm{Mg}$ isotopic ratios include continuum fitting, microturbulence, macroturbulence, identified and unidentified blends. Errors in the model parameters would equally affect the ${ }^{24} \mathrm{MgH},{ }^{25} \mathrm{MgH}$, and ${ }^{26} \mathrm{MgH}$ lines and so the isotopic ratio is quite insensitive to the selected model parameters. Inspection of Figs. 7, 8, 10, and 11 show that even with exquisite signal-to-noise, it can be difficult to discern by eye differences in the syntheses below the level $\mathrm{b} \pm 5$ or $\mathrm{c} \pm 5$ when expressing the ratio as ${ }^{24} \mathrm{Mg}:{ }^{25} \mathrm{Mg}:{ }^{26} \mathrm{Mg}=$ $(100-b-c): b: c$.

The three stars observed separately from the rest of the sample $(702,708$, A88) had lower signal-to-noise. The effect of this lower signal-to-noise can be estimated by considering NGC 6752-mg3. This star was observed with the main sample and also with the three stars. Analysis of the spectrum with inferior signal-to-noise gave an isotopic ratio 79:6:15 which compares favourably with the analysis of the high quality spectrum, 76:9:15.

We focus now upon the two comparison field stars analyzed Shetrone (1996b), HD 103036 and HD 141531. Shetrone's Mg isotopic ratios for HD 103036 and HD 141531 are 94:03:03 and 90:05:05 respectively while we derive 94:00:06 and 91:02:06 respectively. Shetrone's data does not allow a distinction 


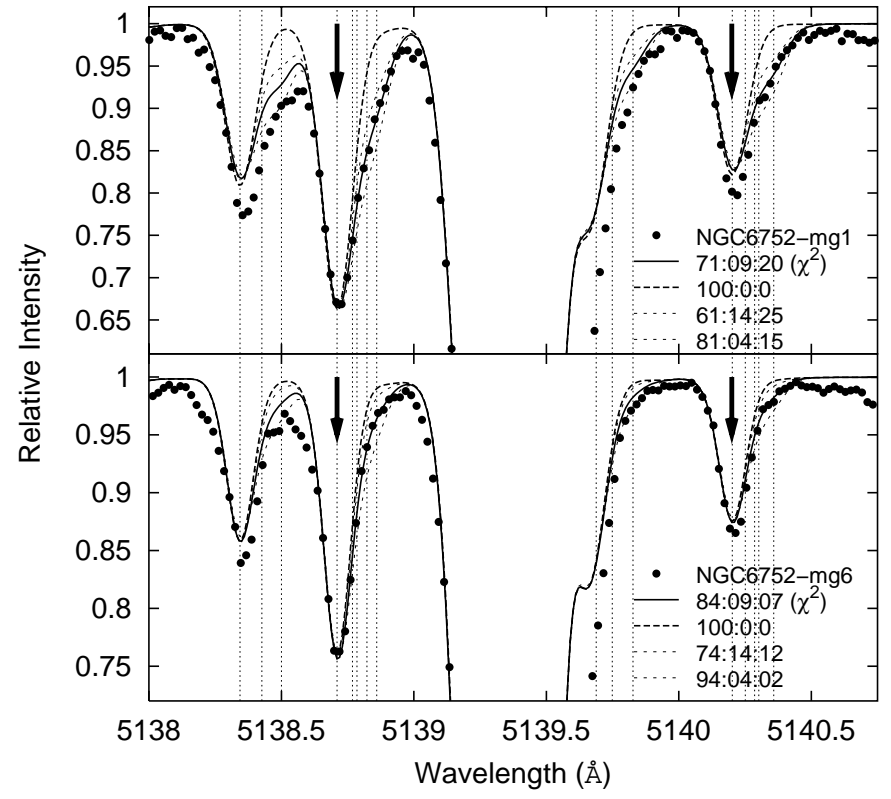

Fig. 8. Spectra of NGC 6752-mg1 (upper) and NGC 6752-mg6 (lower) for 5138.0 to $5140.8 \AA$. The features we are interested in fitting are highlighted by the arrows. The positions of the ${ }^{24} \mathrm{MgH},{ }^{25} \mathrm{MgH}$, and ${ }^{26} \mathrm{MgH}$ lines are indicated by dashed lines. The closed circles represent the observed spectra. The synthetic spectrum generated using the isotopic ratios determined by $\chi^{2}$ analysis is given by the solid line: the ${ }^{24} \mathrm{Mg}:{ }^{25} \mathrm{Mg}:{ }^{26} \mathrm{Mg}$ ratios are given on the figure. Unsatisfactory ratios are plotted as dotted lines.

between ${ }^{25} \mathrm{Mg}$ and ${ }^{26} \mathrm{Mg}$ and is based upon different $\mathrm{MgH}$ lines. Our data is of higher quality (resolving power and signalto-noise), we separate the contributions of ${ }^{25} \mathrm{Mg}$ from ${ }^{26} \mathrm{Mg}$, and rely upon the 3 recommended features used in the Gay \& Lambert (2000) analysis.

\section{Magnesium isotopes and light element abundances}

\subsection{Observed correlations and implications}

Correlations between $\mathrm{O}, \mathrm{Na}, \mathrm{Mg}$ and $\mathrm{Al}$ were presented in Fig. 4. Here we extend the search to correlations between the $\mathrm{Mg}$ isotopic abundances and the light element abundances. Figure 12 shows the $\mathrm{Mg}$ isotopic abundances and their relation to the $\mathrm{Al}$ abundance. The isotopic abundances are obtained by combining the $\mathrm{Mg}$ elemental abundance from the $\mathrm{Mg}$ I lines with the isotopic ratios from the $\mathrm{MgH}$ lines. Isotopic wavelength shifts for the $\mathrm{Mg}$ I and $\mathrm{Al}$ I lines are so small that these provide the respective elemental abundances. We note in anticipation of discussion of the nucleosynthesis proposals that this means that the $\mathrm{Al}$ abundance may be the sum of the stable isotope ${ }^{27} \mathrm{Al}$ and the radioactive isotope ${ }^{26} \mathrm{Al}$ with a half-life of $7 \times 10^{5}$ years.

It is clear that the ${ }^{26} \mathrm{Mg}$ abundance is well correlated with the $\mathrm{Al}$ abundance (Fig. 12 - bottom panel). The total spread in the ${ }^{26} \mathrm{Mg}$ abundance is about a factor of 4 , a range much greater than the measurement uncertainties. In contrast, the ${ }^{25} \mathrm{Mg}$ abundance appears to be constant over the 1.1 dex range in the Al abundance. The ${ }^{24} \mathrm{Mg}$ abundance declines slightly with

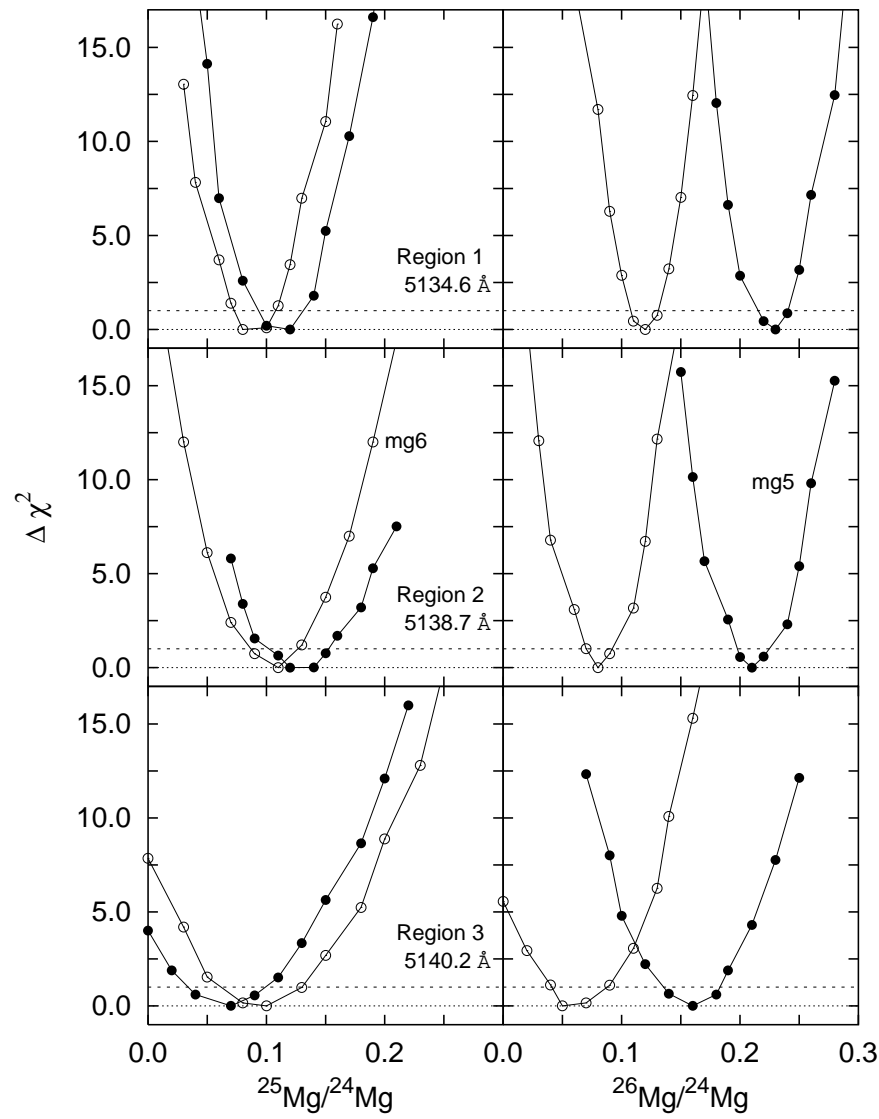

Fig. 9. Variation of the $\chi^{2}$ fit for NGC 6752-mg5 (closed circles) and NGC 6752-mg6 (open circles). The left panels show the variation of $\chi^{2}$ for various values of ${ }^{25} \mathrm{Mg} /{ }^{24} \mathrm{Mg}$ while the right panels show the variation of $\chi^{2}$ for various values of ${ }^{26} \mathrm{Mg} /{ }^{24} \mathrm{Mg}$. The upper panels show the variation of $\chi^{2}$ for Region 1 (5134.6 $\AA$ ), the middle panels represents Region 2 (5138.7 $\AA$ ), and the lower panels represents Region $3(5140.2 \AA)$. The line indicating $1 \sigma\left(\Delta \chi^{2}=1\right)$ errors is shown.

increasing $\mathrm{Al}$ abundance. Our results are consistent with those reported by Shetrone (1996b) for M 13 and by Shetrone (1998) for NGC 6752. Shetrone found large amounts of ${ }^{25} \mathrm{Mg}+{ }^{26} \mathrm{Mg}$ relative to ${ }^{24} \mathrm{Mg}$ in stars with the highest $\mathrm{Al}$ and lowest $\mathrm{Mg}$ abundances. The abundance of ${ }^{24} \mathrm{Mg}$ decreased as $\mathrm{Al}$ increased while ${ }^{25} \mathrm{Mg}+{ }^{26} \mathrm{Mg}$ was not correlated with Al. Unfortunately, Shetrone's spectra were not of sufficient quality to allow for a distinction between ${ }^{25} \mathrm{Mg}$ and ${ }^{26} \mathrm{Mg}$.

In the evolutionary versus primordial debate on the origins of the star-to-star abundance variations, the presence or absence of a correlation of an abundance with a red giant's luminosity may be a powerful debating point. Figure 13 plots the $\mathrm{O}, \mathrm{Na}$, $\mathrm{Mg}$, and $\mathrm{Al}$ abundances versus $T_{\text {eff }}$, a surrogate for luminosity for the red giants. The relation between the $\mathrm{Mg}$ isotopic abundances and $T_{\text {eff }}$ is shown in Fig. 14. In each panel, our red giants are represented by one of the three different symbols: the open circles identify the four stars with the highest ${ }^{26} \mathrm{Mg}$ abundance, the open squares the two stars with the lowest $\mathrm{Al}$ abundance, the filled circles all other stars. The open circles and open squares represent stars at opposite ends of the abundance variation. We also show in Fig. 13 results for the Grundahl et al. (2002) sample of lower luminosity red giants. As noted earlier, 


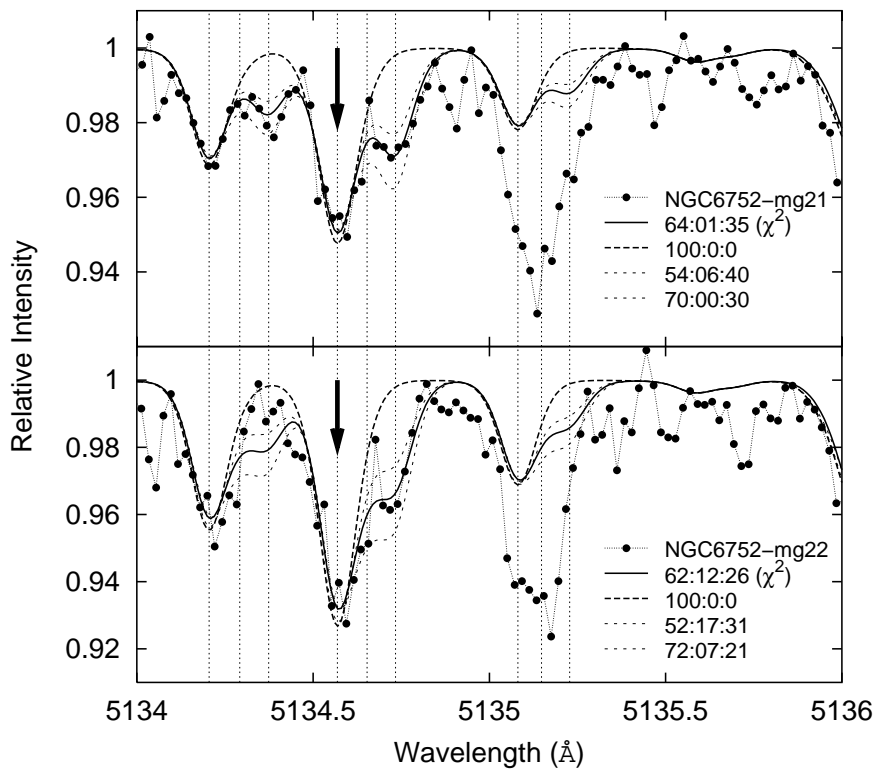

Fig. 10. Spectra of NGC 6752-mg21 (upper) and NGC 6752-mg22 (lower) from 5134 to $5136 \AA$ showing the $\mathrm{MgH}$ lines of interest. The closed circles represent the observed spectra. Although rather weak, the $\mathrm{MgH}$ lines show remarkable asymmetries. In both panels we plot the best fit (solid line) along with unsatisfactory ratios (dotted lines). The asymmetries seen in the $\mathrm{MgH}$ lines are not present in atomic lines.

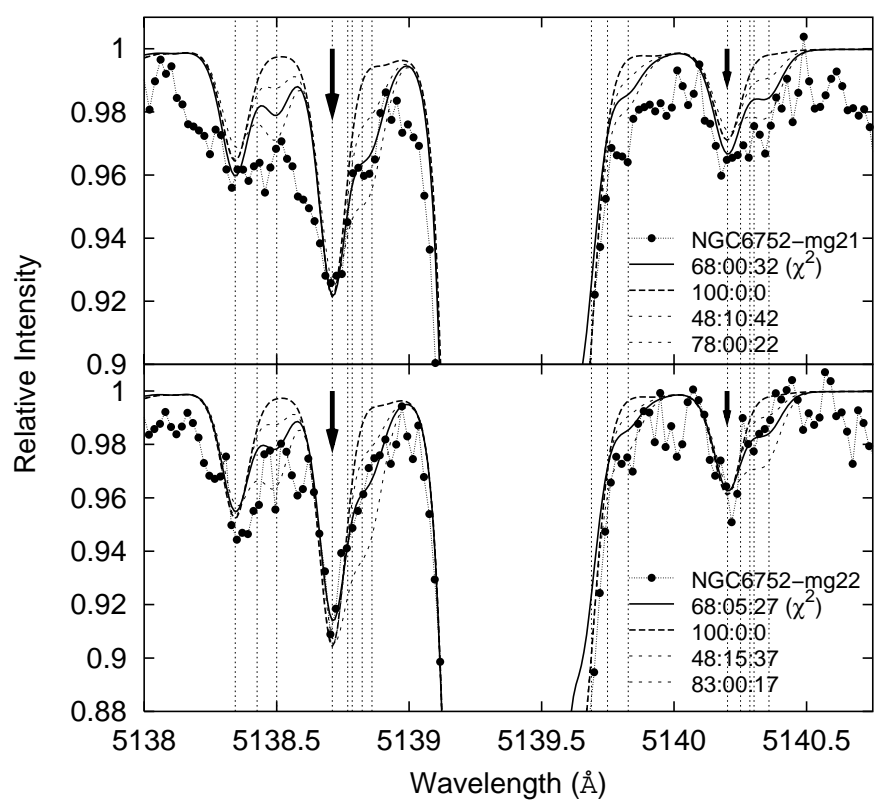

Fig. 11. Spectra of NGC 6752-mg21 (upper) and NGC 6752-mg22 (lower) from 5138 to $5141 \AA$ showing the $\mathrm{MgH}$ lines of interest. The closed circles represent the observed spectra. In both panels, we plot the best fit (solid line) along with unsatisfactory ratios (dotted lines).

the ranges of the elemental abundances agree well with those for our more luminous giants. There is no evidence in Figs. 13 and 14 for a correlation between an abundance and luminosity (i.e., $T_{\text {eff }}$ ). In particular, the four ${ }^{26} \mathrm{Mg}$ richest giants occur at the extremities of the $T_{\text {eff }}$-range, and the least luminous stars of our sample present the greatest spread in abundances.

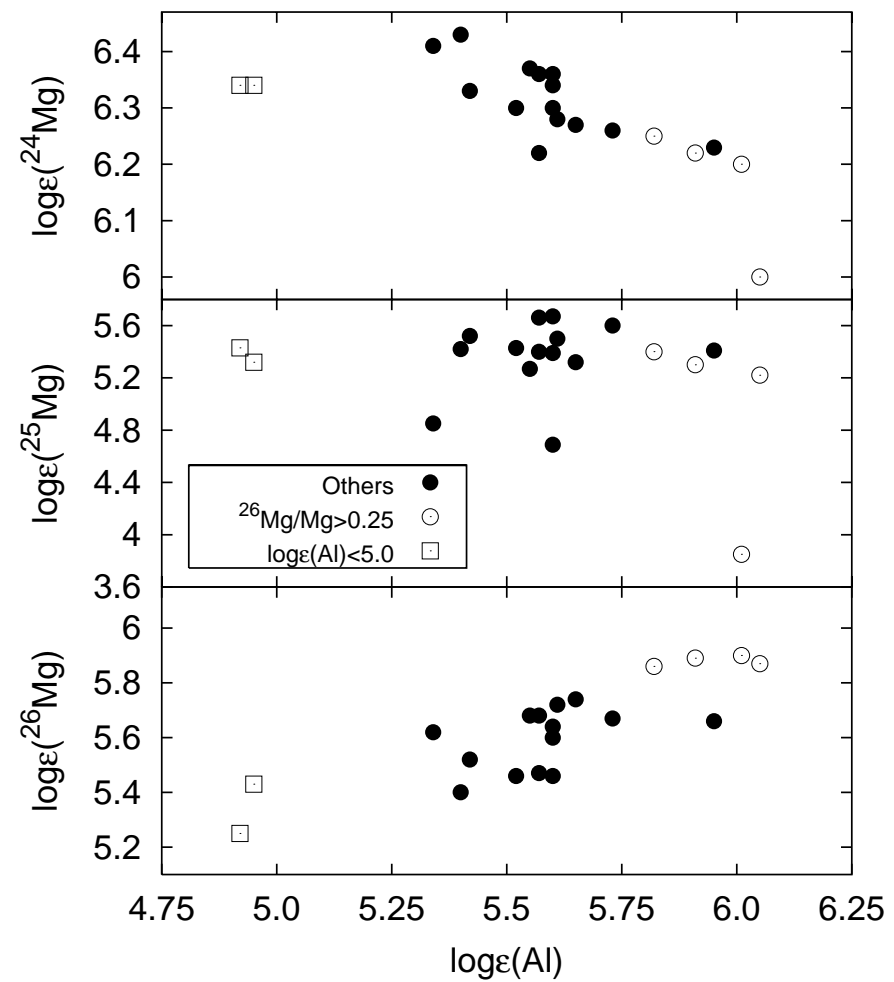

Fig. 12. The abundances of ${ }^{24} \mathrm{Mg},{ }^{25} \mathrm{Mg}$, and ${ }^{26} \mathrm{Mg}$ versus $\mathrm{Al}$. The open circles represent the 4 stars with ${ }^{26} \mathrm{Mg} / \mathrm{Mg}>0.25$ (NGC 6752$\mathrm{mg} 0,21,22,702)$, the open boxes represent stars with $\log \epsilon(\mathrm{Al})<5.0$, and the closed circles are the remaining stars. There is an anticorrelation of ${ }^{24} \mathrm{Mg}$ with $\mathrm{Al}$ and a correlation of ${ }^{26} \mathrm{Mg}$ with $\mathrm{Al}$.

Grundahl et al.'s collection of stars may be subdivided at the bump on the red giant branch. Stars less luminous than the bump show lithium in their atmospheres but stars more luminous than the bump do not; shallow convective mixing dilutes lithium by at least a factor of 10 from a maximum pre-bump abundance of $\log \epsilon(\mathrm{Li}) \sim 1.0$. (Note: we use the term "shallow convective mixing" to refer to mixing responsible for changing $\mathrm{C}, \mathrm{N}$, and $\mathrm{Li}$ abundances and "deep mixing" to refer to the mixing responsible for changing the $\mathrm{O}, \mathrm{Na}, \mathrm{Mg}$, and $\mathrm{Al}$ abundances.) The abundance variations ( $\mathrm{O}, \mathrm{Na}, \mathrm{Mg}$, and $\mathrm{Al})$ are the same in giants more or less luminous than the bump. This result shows that the abundance variations do not reside in a thin layer of accreted material but are present throughout all of the material swallowed by the convective envelope.

Figures 13 and 14 do not support a view that the spread in $\mathrm{O}-\mathrm{Na}-\mathrm{Mg}-\mathrm{Al}$ and $\mathrm{Mg}$ isotopic abundances arise primarily from internal processing and deep mixing with these giants. In contrast to the luminosity dependent $\mathrm{C}$ abundance (Suntzeff \& Smith 1991), there is no systematic dependence in these Figures for any abundance on luminosity (i.e. $T_{\text {eff }}$ ). Anticipating the remarks on the dredge-up of H-burning products, the decline of the $\mathrm{C}$ abundance with increasing luminosity should, if deep mixing is effective, be accompanied by a decline of the $\mathrm{O}$ and $\mathrm{Mg}$ abundances and an increase of the $\mathrm{Na}$ and $\mathrm{Al}$ abundances. This is not seen in Fig. 13. This figure shows more clearly than Fig. 4 did that the distribution function of elemental abundances may differ in shape between ours 


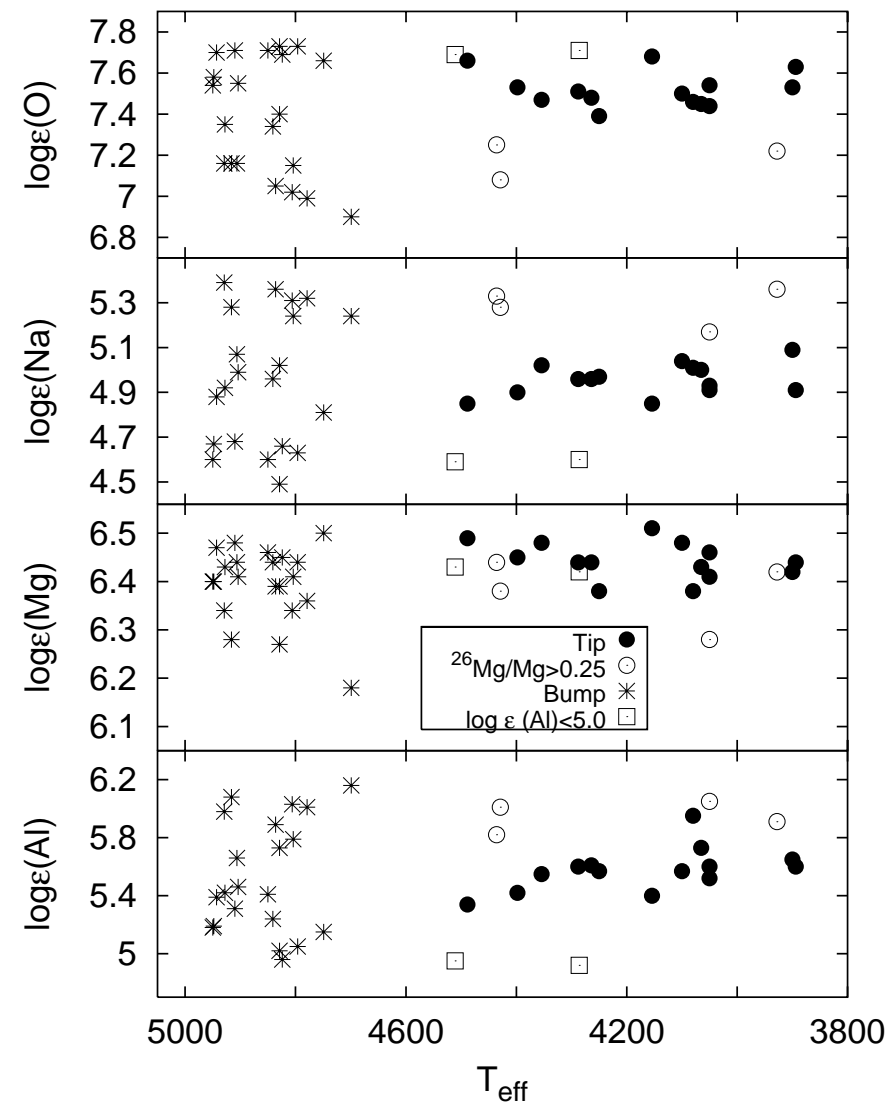

Fig. 13. The elemental abundances of $\mathrm{O}, \mathrm{Na}, \mathrm{Mg}$, and $\mathrm{Al}$ versus $T_{\text {eff }}$. The closed circles represent RGB tip stars from this study, the asterisks represent the Grundahl et al. (2002) RGB bump stars, the open squares represent the 2 stars with $\log \epsilon(\mathrm{Al})<5.0$, and the open circles represent the four RGB tip stars with ${ }^{26} \mathrm{Mg} / \mathrm{Mg}>0.25$ (NGC 6752mg0,21,22,702). Here we take $T_{\text {eff }}$ as a measure of evolutionary status. None of the elemental abundances exhibit a significant correlation with evolutionary status.

and Grundahl et al.'s stars. For example, Grundahl et al.'s stars appear to have relatively more O-poor and Al-poor stars than our sample. This may be a selection effect since all stars near the RGB tip were used whereas the Grundahl et al. stars were chosen using the Strömgren $c_{1}$ index (which Grundahl et al. suspected was tracking the $\mathrm{N}$ abundance) to span the extremes in the abundance distribution. A thorough analysis of a large sample of stars all along the red giant branch is needed to test this tantalising hint.

This result certainly does not exclude the evolutionary scenario entirely. Suppose deep mixing occurs over a narrow luminosity range but only in selected stars. The result would be the appearance of increased star-to-star abundance variations at the critical luminosity. Again, anticipating the discussion of $\mathrm{H}$-burning products, the mean $\mathrm{O}$ and $\mathrm{Mg}$ abundance for the more luminous stars should be lower as the $\mathrm{Na}$ and $\mathrm{Al}$ abundances should be higher than for stars less luminous than the critical luminosity. Such shifts of mean abundances are not evident within our sample or between our and Grundahl et al.'s samples. Of course, the samples are small and there remains the possibility of systematic errors in the abundance errors masking $T_{\text {eff-dependent effects. An absence of a luminosity }}$

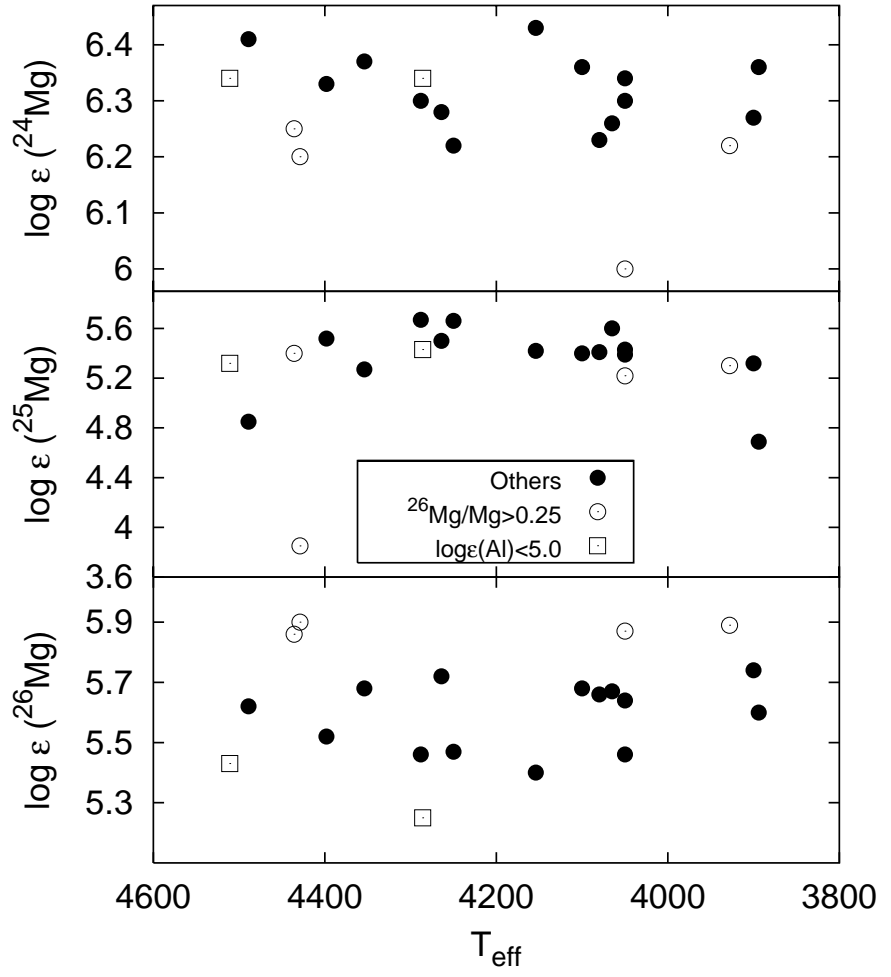

Fig. 14. The abundances of ${ }^{24} \mathrm{Mg},{ }^{25} \mathrm{Mg}$, and ${ }^{26} \mathrm{Mg}$ versus $T_{\text {eff }}$. As in Fig. 13, we take $T_{\text {eff }}$ as a measure of evolutionary status, the open squares represent the 2 stars with $\log \epsilon(\mathrm{Al})<5.0$, and the open circles represent the four stars with ${ }^{26} \mathrm{Mg} / \mathrm{Mg}>0.25$ (NGC 6752-mg0, mg21, $\mathrm{mg} 22$ ). There are no correlations of the $\mathrm{Mg}$ isotopic abundances with evolutionary status.

dependence for the abundances and the presence of similar starto-star abundance variations in the main sequence stars, subgiants, and giants strongly suggests that a primordial not an evolutionary scenario is responsible for the $\mathrm{O}, \mathrm{Na}, \mathrm{Mg}$, and $\mathrm{Al}$ variations.

Clues to plausible scenarios to account for the star-to-star abundance variations are provided by consideration of the extremes of the star-to-star abundance variations. Knowing that field (normal) stars fall at the O-rich, Na-poor, Mg-rich, and Al-poor end of the correlations and anticipating that proton capture reactions can render gas O-poor, Na-rich, and possibly also Mg-poor and Al-rich, we dub the O-rich stars "normal" stars. In the case of the red giants, the normal stars have the composition of the gas from which they formed except for rearrangements of $\mathrm{C}$ and $\mathrm{N}$ introduced by the $\mathrm{CN}$-cycle and shallow convective mixing. According to Fig. 4, the normal stars formed from gas with a composition given by $(\mathrm{O}$, $\mathrm{Na}, \mathrm{Mg}, \mathrm{Al}) \simeq(7.7,4.6,6.45,4.9)$ or equivalently $([\mathrm{O} / \mathrm{Fe}]$, $[\mathrm{Na} / \mathrm{Fe}],[\mathrm{Mg} / \mathrm{Fe}],[\mathrm{Al} / \mathrm{Fe}]) \simeq(0.6,-0.1,0.5,0.0)$. These abundances and those of heavier elements match well those of a field star of a similar metallicity. Extension of this exercise to the $\mathrm{Mg}$ isotopes using Fig. 12 shows that the normal stars have abundances of about 6.35:5.3:5.3 for $\log \epsilon\left({ }^{i} \mathrm{Mg}\right)$ for $i=24,25$, and 26 , respectively, corresponding to the relative abundances ${ }^{24} \mathrm{Mg}:{ }^{25} \mathrm{Mg}:{ }^{26} \mathrm{Mg}=0.8: 0.1: 0.1$.

At the opposite end of the abundance variations to the normal stars are the O-poor stars which we refer to as 
"polluted" stars. The pollution may originate within a star (the evolutionary scenario) or have been imprinted on the star when it was polluted by ejecta from other stars (the primordial scenario). The compositions of stars at the O-poor end of Fig. 4 serve to constrain the composition of the pollutants. A key factor is that the metallicity (iron abundance etc.) is constant across the sample from normal to polluted stars: when observational uncertainties are considered, the iron abundance is independent of (say) the $\mathrm{O}$ abundance to 10 per cent $( \pm 0.04 \mathrm{dex})$ or better. The invariance of the iron abundance constrains both evolutionary and primordial scenarios purporting to account for the polluted stars.

In particular, consider a primordial scenario in which pollutants were contributed by metal-free stars from the generation preceding the present stars. The observations that the cluster's stars have the same metallicity to within about $10 \%$ implies that metal-free pollutants cannot make up more than $10 \%$ of the mass of any star. Then, the maximum underabundance for any element in a polluted star cannot have been greater than the same 10 per cent. This is contrary to the observation that oxygen is underabundant by a factor of 10 in polluted stars. Clearly, the pollutants were not metal-free.

Alternatively, suppose that the pollutants were ejecta from more massive stars of the generation that gave the present stars. In this case, the iron abundance of a polluted star was unaltered by pollution. In the limit that the pollutants contained no oxygen, the factor of 10 decline in the oxygen abundance of the most polluted stars relative to the normal stars implies that the polluted atmospheres are a mix of 9 parts pollutants to one part normal material. The mix shifts in favour of the pollutants in the likely case that the pollutants contain some oxygen; if the oxygen abundance is $10 \%$ of that of normal stars, pollutants dominate the atmosphere of a polluted star. For pollutants with an oxygen abundance greater than $10 \%$ (but less than 100\%), the oxygen abundance of the star falls between that of a normal and the most polluted star.

This discussion shows that, if pollutants and normal gas have the same metallicity, compositions of the most O-poor stars are dominated by pollutants. It then follows that the abundances of elements which are markedly higher in polluted than in normal stars reflect the abundances of those elements in the pollutants. Sodium, ${ }^{26} \mathrm{Mg}$, and $\mathrm{Al}$ are in this category. Figures 4 and 12 then show that the pollutants are a factor of about 0.8 dex enriched in $\mathrm{Na}, 1.0$ dex in $\mathrm{Al}$, and 0.6 dex in ${ }^{26} \mathrm{Mg}$ relative to the normal stars.

In the following sections, we discuss the nucleosynthetic origins of the pollutants in evolutionary and primordial scenarios.

\subsection{Evolutionary scenario}

The evolutionary scenario, as defined here, supposes that the star-to-star differences in $\mathrm{C}$ to $\mathrm{Al}$ abundances result from internal processing and both shallow and deep mixing within the red giant. There is certainly an evolutionary component to the $\mathrm{C}$ and $\mathrm{N}$ abundance variations (Suntzeff \& Smith 1991). Although we have argued that the $\mathrm{O}, \mathrm{Na}, \mathrm{Mg}$, and
Al abundance variations are primarily of a primordial not an evolutionary origin, we comment on the principal ideas incorporated into the theoretical proposals for deep mixing in low mass red giants that appear in discussions of evolutionary scenarios. Deep mixing is synonymous with $\mathrm{H}$-burning at a high temperature.

Hydrogen-burning at high temperatures qualitatively accounts for the abundance variations: the $\mathrm{CN}$-cycle turns $\mathrm{C}$ to $\mathrm{N}$, the $\mathrm{ON}$-cycles convert $\mathrm{O}$ to $\mathrm{N}$, the $\mathrm{NeNa}$-chain converts ${ }^{22} \mathrm{Ne}$ (and ${ }^{20} \mathrm{Ne}$ ) to ${ }^{23} \mathrm{Na}$, and the $\mathrm{MgAl}$-chain may burn magnesium to aluminium. The Coulomb barrier's height increases from $\mathrm{C}$ to $\mathrm{Mg}$ and, thus, the temperature required for efficient burning increases from $\mathrm{C}$ to $\mathrm{Mg}$. While interior layers with temperatures necessary for consumption of $\mathrm{C}$ may be readily tapped by non-standard models of red giants, major revisions of these models are required to link the convective envelope to deeper hotter layers where $\mathrm{Mg}$ may be converted to Al. In addition, it is in these layers that protons are rapidly consumed with the consequence that material dredged to the surface may alter the $\mathrm{He} / \mathrm{H}$ ratio of the atmosphere. As investigated by D'Antona et al. (2002), He enhancements will greatly affect the HB morphology. A review of red giants and possible deep mixing (evolutionary) scenarios can be found in Salaris et al. (2002). We largely restrict comments to the nucleosynthesis.

Standard models predict a maximum temperature of about $55 \times 10^{6} \mathrm{~K}$ for the H-burning shell for a star at the tip of the red giant branch (Vandenberg et al. 1996). The temperature is lower in less evolved giants. Higher temperatures have been suggested to result from thermal instabilities but the Messenger \& Lattanzio (2002) assessment is that the maximum temperature is likely to be close to estimates from standard models. Several authors have incorporated deep mixing into their red giant models and followed the change of surface abundances. The sense of the changes may be obtained from calculations of $\mathrm{H}$-burning by the simultaneous running of the CNO-cycles, the $\mathrm{Ne}-\mathrm{Na}$ and the $\mathrm{Mg}-\mathrm{Al}$ chains in a layer of uniform temperature and density reported by Langer \& Hoffman (1995). Results were given for $T=40 \times 10^{6} \mathrm{~K}$, a temperature characteristic of the H-burning shell, and several initial conditions. Reduction of the $\mathrm{O}$ abundance to its equilibrium abundance for CNO-cycling occurs before $\mathrm{H}$ is seriously depleted. Sodium enrichment is dependent on the conversion of ${ }^{22} \mathrm{Ne}$ to ${ }^{23} \mathrm{Na}$. Plausible values for the "unknown" abundance of ${ }^{22} \mathrm{Ne}$ allow the observed $\mathrm{Na}$ abundance to be achieved before $\mathrm{H}$ exhaustion. The abundance changes in the atmosphere depend on the fraction of material in the convective envelope exposed to the high temperatures. If a large fraction is exposed, the decrease in the $\mathrm{O}$ abundance and the increase in the $\mathrm{Na}$ abundance approach the values shown by stars at the low-O and high-Na end of the star-to-star variations (Langer et al. 1993; Langer \& Hoffman 1995).

A problem arises in extending this evolutionary scenario to the $\mathrm{Mg}-\mathrm{Al}$ chain. At $\mathrm{T}=40 \times 10^{6} \mathrm{~K},{ }^{24} \mathrm{Mg}$ is immune to proton capture, and ${ }^{27} \mathrm{Al}$ is produced solely from ${ }^{25} \mathrm{Mg}$ and ${ }^{26} \mathrm{Mg}$ with substantial depletions of these $\mathrm{Mg}$ isotopes occurring before $\mathrm{H}$ exhaustion. Significant amounts of radioactive ${ }^{26} \mathrm{Al}$ (say, ${ }^{26} \mathrm{Al} /{ }^{27} \mathrm{Al} \sim 0.5$ ) are produced, which, after a million years or so, is present as ${ }^{26} \mathrm{Mg}$. Our observed ${ }^{25} \mathrm{Mg}$ abundances are independent of the $\mathrm{Al}$ abundance and not sharply 
declining as predicted (Denissenkov et al. 1998). The ${ }^{26} \mathrm{Mg}$ abundance increases with $\mathrm{Al}$ abundance but the prediction is that it should probably decline. In short, mixing into a $\mathrm{H}$ burning shell at $T \sim 40 \times 10^{6} \mathrm{~K}$ will not account for the observed $\mathrm{Mg}$ isotopic abundances.

By activating proton capture on ${ }^{24} \mathrm{Mg}$, the concentrations of ${ }^{25} \mathrm{Mg}$ and ${ }^{26} \mathrm{Mg}$ can be maintained, and the potential supply of Al increased. Langer et al. (1997) show that in H-burning at $70 \times 10^{6} \mathrm{~K}$ the abundances of ${ }^{25} \mathrm{Mg}$ and ${ }^{26} \mathrm{Mg}$ (relative to ${ }^{24} \mathrm{Mg}$ ) are approximately preserved as the ${ }^{24} \mathrm{Mg}$ is depleted. Langer et al.'s calculations imply a poor fit to the observed ${ }^{26} \mathrm{Mg}-\mathrm{Al}$ abundance trend. Langer et al. (1997) state that observed extreme abundances for M 13, a cluster with similar star-to-star variations to NGC 6752, are achievable if the envelope is a 9 to 1 mix of material exposed severely to $70 \times 10^{6} \mathrm{~K}$ and original material.

The preceding calculations by Langer and colleagues concerned only the nucleosynthesis and did not attempt to explore how mixing to $T \sim 70 \times 10^{6} \mathrm{~K}$ might occur in a red giant. Among a variety of schemes, we mention a model of "flashassisted deep mixing" presented by Fujimoto et al. (1999) and Aikawa et al. (2001) in an attempt to understand the Mg isotopic and Al abundances reported by Shetrone (1996a,b) for M 13. In this model, hydrogen is mixed into the He core to temperatures even exceeding $70 \times 10^{6} \mathrm{~K}$. The authors contend that "our scenario can be distinguished from others by the fact that ${ }^{25} \mathrm{Mg}$ and ${ }^{26} \mathrm{Mg}$ are enriched at the expense of ${ }^{24} \mathrm{Mg}$ ". Detailed predictions are not provided but qualitatively the model for $[\mathrm{Fe} / \mathrm{H}]=-1.6$ may match our results. A potential problem is that the model, like all deep mixing models tapping into very hot layers, brings helium from the core into the envelope and the atmosphere. This helium with that produced by H-burning at high temperatures may change the $\mathrm{He} / \mathrm{H}$ ratio of the atmosphere by a factor of 2 to 3 . This reduces the atmospheric opacity and enhances the strength of iron (and other) lines. An abundance analysis made with the assumption that all stars have the same $\mathrm{He} / \mathrm{H}$ ratio would then likely result in a spread in the $\mathrm{Fe} / \mathrm{H}$ ratios dependent on (say) the $\mathrm{Al}$ abundance, unless there are compensating effect. This spread is not seen.

The evolutionary scenario in which abundance changes of the light elements $\mathrm{C}$ to $\mathrm{Al}$ are entirely attributable to internal nucleosynthesis and both shallow and deep mixing in the observed red giants faces major challenges. The foremost and seemingly insuperable challenge is that the star-to-star variation in light element abundances is found amongst stars from the main sequence to the tip of the red giant branch without obvious signs of either a difference in the amplitude of the variations or an onset of changes on the red giant branch associated with the occurrence of deep mixing. Shallow convective mixing affecting conversion of $\mathrm{C}$ to $\mathrm{N}$ evidently occurs (Suntzeff \& Smith 1991). (Our sample is dominated by first ascent giants. A contribution from AGB stars to the evolutionary scenario cannot yet be excluded.) A second challenge is that there is as yet no ab initio requirement for deep mixing to occur down to layers at the temperatures at which the $\mathrm{Mg}-\mathrm{Al}$ chain may run. Even with mixing induced by various artifices, it is not obvious that deep mixing can account for the $\mathrm{Mg}$ isotopic abundances reported here.

\subsection{Primordial scenario}

The primordial scenario considers the star-to-star abundance variations to have been imprinted on the present generation of stars at or after their birth. Given that the range of the variations does not decrease between the main sequence and the red giant phases, the imprinting cannot have been confined to a thin skin of the main sequence star but must have affected the entire main sequence star or a very large fraction of it. While abundances of the light elements $\mathrm{C}-\mathrm{Al}$ show considerable star-tostar variations, the abundances of heavier elements, commonly represented by iron, are highly uniform from star to star with the exception of the $\mathrm{Ba}$ and Eu variations seen in M 15 (Sneden et al. 1997).

Uniformity of the metallicity of stars within a globular cluster has surprisingly evoked far less theoretical attention as measured by published papers than the search for an acceptable deep mixing (evolutionary) scenario. In broad terms (see Cayrel 1986; Brown et al. 1991, 1995; Lin \& Murray 1996; Parmentier et al. 1999; Nakasato et al. 2000), it is envisaged that a metal-free (or very metal-poor) cloud of gas forms a first generation of stars. The massive stars of this generation explode as supernovae. Their metal-enriched ejecta mix with the remaining metal-poor cluster gas to form a gaseous shell around the former star cluster. Order of magnitude estimates place the metallicity of the shell in the range exhibited by the Galactic globular clusters. Similarly, it is argued that the ejecta will be so well mixed into the primordial gas that the shell will have a uniform composition. This argument was likely driven by the knowledge that present cluster stars have a common metallicity. It is assumed with or without supporting justification that the intermediate and low mass first generation stars (i.e., stars not dying as supernovae) are quickly ejected from the cluster. Certainly, there is no observational evidence now for a population of lower metallicity stars. The present or second generation of stars is considered to form in the shell.

A successful primordial scenario should not only account for the star-to-star variations of light element abundances but also explain the composition of the normal stars. These stars have a composition that resembles that of field stars of the same metallicity. Numerous attempts to explain the compositions of metal-poor stars by modelling the Galaxy's chemical evolution (e.g., Alibés et al. 2001) suggest that the elemental abundances of NGC 6752's "normal" stars betray the signature of very metal-poor material contaminated by ejecta from very metal-poor massive stars dying as type II supernovae. There is, however, at least one exception to this conclusion, an exception revealed by our observations for the first time. Models of very metal-poor supernovae predict very small yields of the two neutron-rich $\mathrm{Mg}$ isotopes, say relative abundances ${ }^{24} \mathrm{Mg}:{ }^{25} \mathrm{Mg}:{ }^{26} \mathrm{Mg}=0.98: 0.01: 0.01$ (Timmes et al. 1995). This is in sharp contrast to Fig. 12 where the isotopic abundances of the normal stars are about ${ }^{24} \mathrm{Mg}:{ }^{25} \mathrm{Mg}:{ }^{26} \mathrm{Mg}=0.8: 0.1: 0.1$. This disparity between observed and predicted $\mathrm{Mg}$ isotopic ratios suggests that either the predicted yields from supernovae are in error or there was an additional source, one rich in ${ }^{25} \mathrm{Mg}$ and ${ }^{26} \mathrm{Mg}$, which contributed along with the supernovae to the contamination of the very metal-poor gas. 
The additional source may be intermediate mass (IM) AGB stars belonging to the first generation of stars. These stars, with lifetimes only slightly longer than those of the lowest mass stars to explode as supernovae, eject gas at low velocities. Since these $Z=0$ IM-AGBs cannot synthesise iron, their ejecta must be thoroughly mixed throughout the cluster's gas before the present (second generation) stars formed in order that these stars exhibit a common iron abundance. Evolution of and nucleosynthesis by metal-free $(Z=0)$ IM-AGB stars has been examined theoretically by Marigo et al. (2001), Chieffi et al. (2001), and Siess et al. (2002). Of these studies, only that by Siess et al. has considered surface (i.e., ejecta) abundances of the $\mathrm{Mg}$ isotopes. Synthesis of ${ }^{25} \mathrm{Mg}$ and ${ }^{26} \mathrm{Mg}$ but not ${ }^{24} \mathrm{Mg}$ occurs primarily from $\alpha$-captures on ${ }^{22} \mathrm{Ne}$ with the latter produced from $3 \alpha$-processed ${ }^{12} \mathrm{C}$, proton-captures on the ${ }^{12} \mathrm{C}$ producing ${ }^{14} \mathrm{~N}$ which after two $\alpha$ captures becomes ${ }^{22} \mathrm{Ne}$. The key to efficient synthesis of the Mg isotopes is that the AGB stars near their terminal luminosity experience protoncaptures at the base of the convective envelope, the so called hot bottomed convective envelope. A back-of-the-envelope calculation shows that through a combination of an unexceptional initial mass function and the yields predicted by Siess et al., the IM-AGBs can supply sufficient ${ }^{25} \mathrm{Mg}$ and ${ }^{26} \mathrm{Mg}$ to raise the low abundances of ${ }^{25} \mathrm{Mg}$ and ${ }^{26} \mathrm{Mg}$ provided by the supernovae to the much higher levels observed in the normal stars. The IMAGBs may also account for much of the $\mathrm{Na}$ and possibly the Al seen in the normal stars. These hot bottomed AGB stars have envelopes rich in ${ }^{14} \mathrm{~N}$. Supernovae seem incapable of enriching $Z=0$ gas to the observed ${ }^{14} \mathrm{~N}$ abundance of the normal second generation stars (Denissenkov et al. 1997; Alibés et al. 2001). The IM-AGB ejecta may also account for the ${ }^{14} \mathrm{~N}$ abundance.

The origin of the pollutants causing the star-to-star abundance variations for light elements in the present stars cannot be the first-generation IM-AGB stars - see Sect. 6.1. Second generation IM-AGBs are eligible sources of the pollutants. Cottrell \& Da Costa (1981), who discovered the $\mathrm{Na}$ and Al abundance variations in globular cluster giants from spectra of NGC 6752's giants, suggested IM-AGBs as sources and their He-burning shell as the site for the synthesis of $\mathrm{Na}$ and Al: ${ }^{22} \mathrm{Ne}(\alpha, n)^{25} \mathrm{Mg}$ and ${ }^{22} \mathrm{Ne}(\alpha, \gamma){ }^{26} \mathrm{Mg}$ is followed by neutron captures on ${ }^{22} \mathrm{Ne}$ and the $\mathrm{Mg}$ isotopes to make $\mathrm{Na}$ and $\mathrm{Al}$, respectively. A traditional s-process leading to heavy elements is inhibited by loss of neutrons to nuclei lighter than iron. This theoretical conclusion is consistent with the fact that Na-rich and Al-rich cluster giants are not $s$-process enriched (e.g., see Ivans et al. 2001). Association of $\mathrm{Na}$ and $\mathrm{Al}$ synthesis with the He-burning shell ran into difficulties when it was subsequently shown that the $\mathrm{Na}$ and Al-rich stars were depleted in oxygen (e.g., see review by Suntzeff 1993) because ${ }^{16} \mathrm{O}$ is also synthesised in the He-burning shell. This difficulty was alleviated once it was recognized that metal-poor IM-AGBs develop a hot bottomed convective envelope capable of running the H-burning ON-cycles and burning $\mathrm{O}$ to $\mathrm{N}$. Reductions of the envelope's $\mathrm{O}$ abundance by factors of 10 to 100 are possible depending on the temperatures at the base of the envelope and the total exposure of envelope material to high temperatures. Additional processing of light elements $-\mathrm{Ne}$ to $\mathrm{Al}-$ including participation of ${ }^{24} \mathrm{Mg}$ in the $\mathrm{Mg}-\mathrm{Al}$ chain may occur at the envelope's base. Nucleosynthesis by metal-poor IM-AGBs have been reported by Forestini \& Charbonnel (1997) for $Z=0.005$, Ventura et al. $(2001,2002)$ for $Z=$ $2 \times 10^{-4}$, and Karakas \& Lattanzio (2003, private communication) for $Z=0.004$ and 0.008 . Karakas \& Lattanzio show that low metallicity IM-AGBs can develop an envelope (i.e., subsequently comprising the ejecta) rich in ${ }^{25} \mathrm{Mg}$ and/or ${ }^{26} \mathrm{Mg}$ and Al. (The $Z$ of NGC 6752's stars is a factor of 2 to 3 less than the lowest value considered by Karakas \& Lattanzio.) The impression from Fig. 12 is that the pollutants are rich in ${ }^{26} \mathrm{Mg}$ but not in ${ }^{25} \mathrm{Mg}$. Production of ${ }^{26} \mathrm{Mg}$ without without ${ }^{25} \mathrm{Mg}$ is implausible for the $\mathrm{Mg}-\mathrm{Al}$ chain. What may be happening is that the ${ }^{25} \mathrm{Mg}$ abundance is subject to two effects which balance out: production of ${ }^{25} \mathrm{Mg}$ from ${ }^{24} \mathrm{Mg}$ and loss of ${ }^{25} \mathrm{Mg}$ to ${ }^{26} \mathrm{Mg}$.

It is not sufficient to show that second generation IM-AGBs may have envelopes with a composition closely resembling that inferred above for the pollutants necessary to transform a normal to a polluted star. One must show that a low mass cluster star can accrete sufficient mass of ejecta from the IM-AGBs to be transformed from a normal to a polluted star, and that this transformation is a common occurrence for the very large number of stars in the cluster. Fortunately, Thoul et al.'s (2002) detailed study of the fate of ejecta from IM-AGBs in a globular cluster shows that accretion of intracluster gas may be an efficient process. In the case of NGC 6752, Thoul et al. show that "more than $60 \%$ of the gas ejected by the AGB stars is accreted by the cluster stars" and " $1 M_{\odot}$ stars can accrete an appreciable fraction of their initial mass. The envelopes of those stars will reflect the composition of the intracluster medium rather than the composition of their interior even if a physical mechanism is at work to induce mixing with the deeper layers of the star". At this level of contamination, it is to be expected that the starto-star abundance variations will be very similar for dwarfs and giants despite the deep convective envelope of a giant ${ }^{5}$.

In summary, the second generation IM-AGBs synthesise and eject the needed pollutants into the intracluster medium which the present low mass stars accreted. Normal stars are but slightly contaminated by pollutants. The polluted stars are severely contaminated.

\section{Concluding remarks}

Star-to-star variations of light element $(\mathrm{O}$ to $\mathrm{Al})$ abundances among globular cluster stars are now well documented. For NGC 6752, the cluster where $\mathrm{Na}$ and $\mathrm{Al}$ abundance variations were discovered by Cottrell \& Da Costa (1981), we have extended investigations of abundances for cluster red giants to the isotopic ratios of $\mathrm{Mg}$ to provide measurements of the ratios ${ }^{24} \mathrm{Mg}:{ }^{25} \mathrm{Mg}:{ }^{26} \mathrm{Mg}$ in stars sampling the full range of the abundance variations. These isotopic ratios proved to offer new clues to the chemical evolution of the cluster. In the case of NGC 6752 (and other clusters), there is now strong evidence that the star-to-star variations in the $\mathrm{O}, \mathrm{Na}, \mathrm{Mg}$, and $\mathrm{Al}$ abundances are present in dwarfs, subgiants as well as the giants for

\footnotetext{
5 Mass transfer across a binary system is another way for a low mass star to acquire pollutants. This is the process by which classical Barium and $\mathrm{CH}$ stars in the field are formed.
} 
which the initial discoveries were made. This suggests that the abundance variations arise in the course of the chemical evolution of the cluster.

According to the canonical picture of a globular cluster, the first generation of stars formed from primordial gas of very low metallicity $(Z \simeq 0)$. Supernovae ejecta from this generation mixed with primordial gas to raise the metallicity of the cluster gas. A second generation of stars formed from the cooled enriched gas. Low mass stars of this generation comprise the present cluster members. All first generation stars are presumed to have been ejected at an early time.

Into this simple picture must be woven ideas to account for the star-to-star abundance variations. Current ideas involve the IM-AGB stars and their ejecta which can be put into the intracluster gas on a relatively short timescale. Our novel results for the $\mathrm{Mg}$ isotopic ratios suggest roles for both first and second generation IM-AGBs.

Stars with the highest $\mathrm{O}$ abundance and lowest $\mathrm{Na}$ and $\mathrm{Al}$ abundances (normal stars in our parlance) are considered to have formed from the cluster's gas after the primordial gas had been thoroughly mixed with the supernovae ejecta. Our ${ }^{25} \mathrm{Mg}$ and ${ }^{26} \mathrm{Mg}$ abundances (relative to ${ }^{24} \mathrm{Mg}$ ) are about an order of magnitude higher than predicted for ejecta from $Z=0$ massive stars exploding as supernovae. One way to increase the ${ }^{25} \mathrm{Mg}$ and ${ }^{26} \mathrm{Mg}$ abundances is by contamination of the cluster's gas by ejecta from first generation $Z=0$ IM-AGBs followed by thorough mixing of the ejecta occurring before the onset of star formation that led to the second generation of stars. The ejecta are likely to have contributed not only ${ }^{25} \mathrm{Mg}$ and ${ }^{26} \mathrm{Mg}$ but also a considerable fraction of the $\mathrm{Na}$ and $\mathrm{Al}$ of the normal stars. Second generation stars dwell in the intracluster medium into which the IM-AGBs of this generation eject their envelopes at low velocity. Accretion of this gas by lower mass stars pollutes the stars and introduces the star-to-star light element abundance variations.

This cartoon of an evolutionary scenario demands observational examination. In the case of NGC 6752, the abundances of light elements in AGB stars and, especially, in the mainsequence stars and subgiants should be determined. Apparent systematic abundance differences between the abundances $(\mathrm{O}$, $\mathrm{Na}, \mathrm{Mg}, \mathrm{Al}$, and $\mathrm{Fe}$ ) among giants and those of the main sequence stars and subgiants reported by Gratton et al. (2001) would, if real, present any evolutionary or primordial scenario with a challenge. Of great value would be measurements of the $\mathrm{Mg}$ isotopic ratios for giants of other globular clusters. It will be difficult to push the search to clusters lower in metallicity than NGC 6752 (see Shetrone 1996b) because the $\mathrm{MgH}$ lines will be very weak. Exploration of giants in several clusters of NGC 6752's or higher metallicity is certainly possible with very large telescopes. Not only will it be important to see if other clusters showing a large spread in oxygen abundances (e.g., M 13) show the same dependence of the Mg isotopes on the $\mathrm{Al}$ (or $\mathrm{Na}$ ) abundances, but it will be interesting to determine the isotopic abundances in clusters (e.g., M 71) where there is very little star-to-star variation in the $\mathrm{Al}$ abundance. Measurement of the isotopic $\mathrm{O}$ abundances using the $\mathrm{CO}$ infrared bands should be made. Severe ON-cycling in the hot bottomed envelope of an IM-AGB may produce copious amounts of ${ }^{17} \mathrm{O}:{ }^{17} \mathrm{O} /{ }^{16} \mathrm{O} \sim 0.1$ (Ventura et al. 2001, 2002). These observations hold the promise of unravelling the evolution of the globular clusters and, perhaps, their origin.

Evolutionary paths may differ from cluster to cluster. Some paths may end in partial or complete dissolution of the cluster at early or late times. The clusters' origin and evolution may, as many have speculated, be related to the origin of some halo field stars. In closing, we offer the following two comments on the roles assigned to IM-AGBs and their possible connection to chemical evolution of clusters and to field halo stars.

First, if the early evolution of the cluster is rapid, the second generation of stars may form before the first generation IM-AGBs contaminate the gas with ${ }^{25} \mathrm{Mg}$, and ${ }^{26} \mathrm{Mg}$ (also $\mathrm{N}, \mathrm{Na}$, and $\mathrm{Al}$ ). In this case, the present (second generation) stars will have the low ${ }^{25} \mathrm{Mg}$ and ${ }^{26} \mathrm{Mg}$ abundances expected of supernovae ejecta. Observational pursuit of $\mathrm{Mg}$ isotopes in other globular clusters may test this speculation. Among metalpoor field stars, it is certainly the case that the speculation would account for the fact that some field stars have the very low ${ }^{25} \mathrm{Mg} /{ }^{24} \mathrm{Mg}$ and ${ }^{26} \mathrm{Mg} /{ }^{24} \mathrm{Mg}$ ratios expected for supernovae ejecta but others of a similar metallicity have the higher ratios found here for normal NGC 6752 stars (Gay \& Lambert 2000). Were these stars with the higher ratios formed in and shed from a cluster after the ejecta from the first generation IM-AGBs had been mixed into the gas? Before answering "yes", an alternative possibility needs to be investigated, namely, some or even all of the stars may be mass transfer binaries in which a companion IM-AGB provided the additional ${ }^{25} \mathrm{Mg}$ and ${ }^{26} \mathrm{Mg}$.

Second, field stars do not show the star-to-star abundance variations seen in NGC 6752 and some other globular clusters (Pilachowski et al. 1996; Hanson et al. 1998). This difference implies that the field stars have not come from globular clusters like NGC 6752. Two obvious corollaries to this conclusion are: (i) the field stars did not form in a closed environment conducive to accretion of ejecta from IM-AGBs of the same generation; (ii) clusters like NGC 6572 cannot have provided many field stars.

Acknowledgements. We thank the referee, Judy Cohen, for helpful comments. DY is grateful to Inese Ivans for helpful discussions and insights. DY thanks Peter Höflich for assistance in running the syntheses on a cluster of workstations financed by the John W. Cox-Fund of the Department of Astronomy at the University of Texas. DLL and DY acknowledge support from the Robert A. Welch Foundation of Houston, Texas. FG gratefully acknowledges generous financial support from the Carlsberg foundation. This research has made use of the SIMBAD database, operated at CDS, Strasbourg, France and NASA's Astrophysics Data System.

\section{References}

Aikawa, M., Fujimoto, M. Y., \& Kato, K. 2001, ApJ, 560, 937 Alcaino, G. 1972, A\&A, 16, 220

Alibés, A., Labay, J., \& Canal, R. 2001, A\&A, 370, 1103

Alonso, A., Arribas, S., \& Martínez-Roger, C. 1999, A\&AS, 140, 261

Bell, R. A., Hesser, J. E., \& Cannon, R. D. 1983, ApJ, 269, 580

Bevington, P. R., \& Robinson, D. K. 1992, Data reduction and error analysis for the physical sciences 2nd ed. (New York: McGrawHill)

Briley, M. M., Cohen, J. G., \& Stetson, P. B. 2002, ApJ, 579, L17 
Brown, J. A., \& Wallerstein, G. 1989, AJ, 98, 1643

Brown, J. H., Burkert, A., \& Truran, J. W. 1991, ApJ, 376, 115

Brown, J. H., Burkert, A., \& Truran, J. W. 1995, ApJ, 440, 666

Buonanno, R., Caloi, V., Castellani, V., et al. 1986, A\&AS, 66, 79

Cannon, R. D., Croke, B. F. W., Bell, R. A., Hesser, J. E., \& Stathakis, R. A. 1998, MNRAS, 298, 601

Cannon, R. D., Da Costa, G. S., Norris, J. E., Stanford, L., \& Croke, B. 2002 [astro-ph/0210324]

Carretta, E., \& Gratton, R. G. 1997, A\&AS, 121, 95

Cayrel, R. 1986, A\&A, 168, 81

Chieffi, A., Domínguez, I., Limongi, M., \& Straniero, O. 2001, ApJ, 554,1159

Cohen, J. G. 1999, AJ, 117, 2434

Cohen, J. G., Briley, M. M., \& Stetson, P. B. 2002, AJ, 123, 2525

Cottrell, P. L., \& Da Costa, G. S. 1981, ApJ, 245, L79

D’Antona, F., Caloi, V., Montalbán, J., Ventura, P., \& Gratton, R. 2002, A\&A, 395, 69

Denissenkov, P. A., Da Costa, G. S., Norris, J. E., \& Weiss, A. 1998, A\&A, 333, 926

Denissenkov, P. A., Weiss, A., \& Wagenhuber, J. 1997, A\&A, 320, 115

Forestini, M., \& Charbonnel, C. 1997, A\&AS, 123, 241

Fujimoto, M. Y., Aikawa, M., \& Kato, K. 1999, ApJ, 519, 733

Gay, P. L., \& Lambert, D. L. 2000, ApJ, 533, 260

Gratton, R. G., Bonifacio, P., Bragaglia, A., et al. 2001, A\&A, 369, 87

Grundahl, F., Briley, M., Nissen, P. E., \& Feltzing, S. 2002, A\&A, 385, L14

Grundahl, F., Catelan, M., Landsman, W. B., Stetson, P. B., \& Andersen, M. I. 1999, ApJ, 524, 242

Hanson, R. B., Sneden, C., Kraft, R. P., \& Fulbright, J. 1998, AJ, 116, 1286

Harris, W. E. 1996, AJ, 112, 1487

Hesser, J. E. 1978, ApJ, 223, L117

Hesser, J. E., \& Bell, R. A. 1980, ApJ, 238, L149

Holweger, H., \& Müller, E. A. 1974, Sol. Phys., 39, 19

Ivans, I. I., Kraft, R. P., Sneden, C., et al. 2001, AJ, 122, 1438

Kraft, R. P., \& Ivans, I. I. 2003, PASP, 115, 143

Kraft, R. P., Sneden, C., Smith, G. H., et al. 1997, AJ, 113, 279

Kurucz, R. 1993, ATLAS9 Stellar Atmosphere Programs and 2 km/s grid. Kurucz CD-ROM No. 13. Cambridge, Mass.: Smithsonian Astrophysical Observatory, 13

Kurucz, R. L., Furenlid, I., \& Brault, J. 1984, Solar flux atlas from 296 to 1300 NM (National Solar Observatory Atlas, Sunspot, New Mexico: National Solar Observatory)

Lambert, D. L., Heath, J. E., Lemke, M., \& Drake, J. 1996, ApJS, 103, 183

Langer, G. E., Hoffman, R., \& Sneden, C. 1993, PASP, 105, 301

Langer, G. E., \& Hoffman, R. D. 1995, PASP, 107, 1177
Langer, G. E., Hoffman, R. E., \& Zaidins, C. S. 1997, PASP, 109, 244 Lehnert, M. D., Bell, R. A., \& Cohen, J. G. 1991, ApJ, 367, 514

Lin, D. N. C., \& Murray, S. D. 1996, in Dynamical Evolution of Star Clusters: Confrontation of Theory and Observations, IAU Symp., 174, 283

Marigo, P., Girardi, L., Chiosi, C., \& Wood, P. R. 2001, A\&A, 371, 152

McWilliam, A., \& Lambert, D. L. 1988, MNRAS, 230, 573

Messenger, B. B., \& Lattanzio, J. C. 2002, MNRAS, 331, 684

Minniti, D., Geisler, D., Peterson, R. C., \& Claria, J. J. 1993, ApJ, 413, 548

Minniti, D., Peterson, R. C., Geisler, D., \& Claria, J. J. 1996, ApJ, 470, 953

Nakasato, N., Mori, M., \& Nomoto, K. 2000, ApJ, 535, 776

Nissen, P. E., Asplund, M., Hill, V., \& D'Odorico, S. 2000, A\&A, 357, L49

Nissen, P. E., Lambert, D. L., Primas, F., \& Smith, V. V. 1999, A\&A, 348, 211

Norris, J., Cottrell, P. L., Freeman, K. C., \& Da Costa, G. S. 1981, ApJ, 244, 205

Norris, J. E., \& Da Costa, G. S. 1995, ApJ, 441, L81

Parmentier, G., Jehin, E., Magain, P., et al. 1999, A\&A, 352, 138

Penny, A. J., \& Dickens, R. J. 1986, MNRAS, 220, 845

Pilachowski, C. A., Sneden, C., \& Kraft, R. P. 1996, AJ, 111, 1689

Ramírez, S. V., \& Cohen, J. G. 2003, AJ, 125, 224

Salaris, M., Cassisi, S., \& Weiss, A. 2002, PASP, 114, 375

Shetrone, M. D. 1996a, AJ, 112, 1517

Shetrone, M. D. 1996b, AJ, 112, 2639

Shetrone, M. D. 1998, in Fundamental Stellar Properties, IAU Symp., 189,158

Siess, L., Livio, M., \& Lattanzio, J. 2002, ApJ, 570, 329

Smith, V. V., \& Suntzeff, N. B. 1989, AJ, 97, 1699

Sneden, C. 1973, ApJ, 184, 839

Sneden, C., Kraft, R. P., Shetrone, M. D., et al. 1997, AJ, 114, 1964

Suntzeff, N. 1993, in The Globular Cluster-Galaxy Connection, ASP Conf. Ser., 48, 167

Suntzeff, N. B., \& Smith, V. V. 1991, ApJ, 381, 160

Thoul, A., Jorissen, A., Goriely, S., et al. 2002, A\&A, 383, 491

Timmes, F. X., Woosley, S. E., \& Weaver, T. A. 1995, ApJS, 98, 617

Tomkin, J., \& Lambert, D. L. 1980, ApJ, 235, 925

Vandenberg, D. A., Stetson, P. B., \& Bolte, M. 1996, ARA\&A, 34, 461

VandenBerg, D. A., Swenson, F. J., Rogers, F. J., Iglesias, C. A., \& Alexander, D. R. 2000, ApJ, 532, 430

Ventura, P., D’Antona, F., \& Mazzitelli, I. 2002, A\&A, 393, 215

Ventura, P., D’Antona, F., Mazzitelli, I., \& Gratton, R. 2001, ApJ, 550, L65

Zinn, R., \& West, M. J. 1984, ApJS, 55, 45 